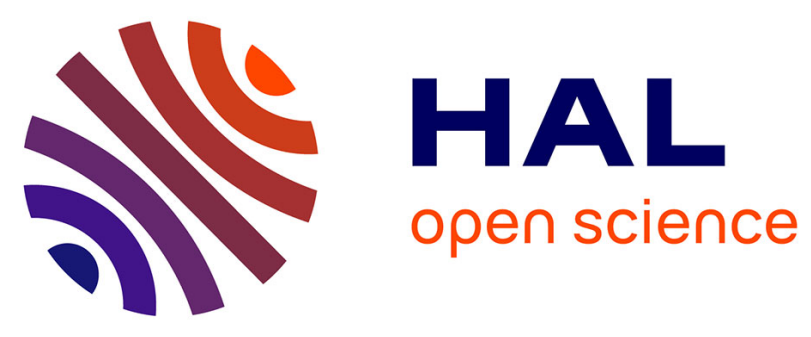

\title{
Characterization of piezoelectric composites with mechanical and electrical imperfect contacts
}

\author{
Reinaldo Rodriguez-Ramos, Raul Guinovart-Diaz, Juan C Lopez-Realpozo, \\ Julian Bravo-Castillero, Federico J Sabina, Frédéric Lebon, Serge Dumont, \\ Mathias Wurkner, Harald Berger, Ulrich Gabbert
}

\section{To cite this version:}

Reinaldo Rodriguez-Ramos, Raul Guinovart-Diaz, Juan C Lopez-Realpozo, Julian Bravo-Castillero, Federico J Sabina, et al.. Characterization of piezoelectric composites with mechanical and electrical imperfect contacts. Journal of Composite Materials, 2016, 50 (12), pp.1603-1625. $10.1177 / 0021998315594681$. hal-01313872

\section{HAL Id: hal-01313872 \\ https://hal.science/hal-01313872}

Submitted on 3 Jul 2018

HAL is a multi-disciplinary open access archive for the deposit and dissemination of scientific research documents, whether they are published or not. The documents may come from teaching and research institutions in France or abroad, or from public or private research centers.
L'archive ouverte pluridisciplinaire HAL, est destinée au dépôt et à la diffusion de documents scientifiques de niveau recherche, publiés ou non, émanant des établissements d'enseignement et de recherche français ou étrangers, des laboratoires publics ou privés. 


\title{
Characterization of piezoelectric composites with mechanical and electrical imperfect contacts
}

\author{
Reinaldo Rodriguez-Ramos', Raul Guinovart-Diaz', \\ Juan C Lopez-Realpozo', Julian Bravo-Castillero', \\ Federico J Sabina ${ }^{2}$, Frederic Lebon ${ }^{3}$, Serge Dumont ${ }^{3}$, \\ Mathias Würkner ${ }^{4}$, Harald Berger ${ }^{4}$ and Ulrich Gabbert ${ }^{4}$
}

\begin{abstract}
The aim of the present work is to study the influence of the mechanical and electrical imperfections in reinforced piezoelectric composite materials with unidirectional cylindrical fibers periodically distributed in rhombic cells under mechanical and electrical imperfect contacts. The behavior of the composites is studied through two approaches: the two-scale asymptotic homogenization method and the finite element method. The asymptotic homogenization method is applied to a two-phase composite with mechanical and electrical imperfect contacts and to a three-phase composite with perfect contact in the interphase. The constituents of the composite are homogeneous piezoelectric materials with transversely isotropic properties. The local problems are formulated for the spring-capacitor and three-phase models by the asymptotic homogenization method. The solution of each plane local problem is found using potential methods of a complex variable and the properties of doubly periodic Weierstrass elliptic functions. Closed-form formulae are obtained for the effective properties of the composites with both types of imperfect contacts and different configuration of the cells. The finite element method is implemented for analysis of piezoelectric composite materials with unidirectional cylindrical fibers periodically distributed in rhombic cells under mechanical and electrical imperfect contacts. Some numerical examples are given under the presence of both imperfect contacts and different arrangement of the cells. Comparisons between the numerical results reported by asymptotic homogenization method and finite element method are provided.
\end{abstract}

\section{Keywords}

Piezoelectric composites, interfacial bonding, imperfect contact, asymptotic homogenization, finite element method

\section{Introduction}

The study of contact phenomena and the modeling of interfaces between two solids gain special importance in the effective property determination of composite media. This work is motivated by the interest to study the influence of imperfect contact over the effective piezoelectric response. Many multi-scale approaches are associated with composites where perfect interface conditions are assumed. However, many results show that local or partial debonding on interfaces is a rule rather than an exception in materials such as reinforced composites. $^{1-5}$ The existence of a stiff region is only an idealization of the complex phenomenon that occurs in the interface where a transition zone (interphase)
'Facultad de Matemática y Computación, Universidad de la Habana, San Lázaro y L, Vedado, Habana 4, Cuba

${ }^{2}$ Instituto de Investigaciones en Matemáticas Aplicadas y en Sistemas, Universidad Nacional Autónoma de México, México D.F., México

${ }^{3}$ Laboratoire de Mécanique et d'Acoustique, Université Aix-Marseille, CNRS, Centrale Marseille, France

${ }^{4}$ Institute of Mechanics, University of Magdeburg, Universitätsplatz 2, Magdeburg, Germany

\section{Corresponding author:}

Harald Berger, Institute of Mechanics, University of Magdeburg, Universitätsplatz 2, D-39106 Magdeburg, Germany.

Email: harald.berger@ovgu.de 
between both components is omnipresent. This third phase is the result of the material fabrication process (chemical treatments of surfaces, resin crystallization). Various approaches have been used, where the bond between the components is modeled by an interphase with specified thickness and elastic, piezoelectric and dielectric properties. We can find different reports about elastic composite. ${ }^{6-8}$ In most piezoelectric composites, the adhesion is not perfect. There are many types of imperfect boundary, in particular, in this work mechanical and electrical imperfect contacts are considered, which means that the continuity condition for the stresses is satisfied but not the continuity conditions for the mechanical displacement and furthermore the continuity condition for the electric displacement field is satisfied but the continuity condition for the electric potential is not fulfilled. The mechanical imperfect contact is considered when the stresses are proportional to the mechanical displacement jump and the electrical imperfect contact is modeled, as the electric displacement field is proportional to the electric potential jump in the composite. This type of mechanical imperfect contact can be interpreted as the presence of springs between both components of the composite, which is usually named in the literature as "mechanical imperfect contact, spring type". In the same way, the electrical imperfect contact is idealized as the existence of a capacitor between both components, which creates a difference in the electric potential between the components of the composite. This approach has been employed in some articles. ${ }^{9-12}$ It is noteworthy to mention that the interface failure in the realm of elastic materials occurs in the form of normal debonding, inplane sliding, out-of-plane sliding or any combination of these types. In other words, imperfection takes place only in the form of displacement discontinuities. However, in the realm of piezoelectric materials, due to their intrinsic coupled electro-mechanical behavior, there are induced electric charges, even if the loading is only mechanical. Therefore, a legitimate question is whether the existence of an interface electric barrier has any effects on the induced electro-mechanical fields. In the present work, it is proposed to model such barriers as the electric capacitors. A capacitor (also called condenser) is produced when two electrical charged plates are separated by a dielectric or insulator. This scenario can readily be modeled with a distribution of electric capacitors along the interface. The degree of the electrical imperfection is directly linked to the degree of discontinuity in the electric potential function at the interface. The capacitors capacitance determines the amount of the electrical imperfection. The interface is a complete electric barrier when the capacitance is equal to zero. As the capacitance increases the electrical imperfection level decreases, and the perfect electrical interface prevails as the capacitance approaches infinity.

The aim of this paper is to point out the role of the mechanical and electrical imperfect interfaces on the elastic, piezoelectric and dielectric properties of piezoelectric composite materials. In particular, the two-scale asymptotic homogenization method $(\mathrm{AHM})^{13-15}$ is applied to a micromechanical model with rhombic arrangement of fibers. This work is an extension of previous works, ${ }^{16-18}$ where piezoelectric composites with only mechanical imperfect contact were considered. The present study also differs from the other investigations, ${ }^{17,19-21}$ because not only the mechanical imperfect contact (spring model) is considered in the electro-elastic composites but also the combination of mechanical and electrical (springcapacitor) imperfections is examined. It may look natural, that the mechanical weakening of the interface (due to delamination, decohesion, etc.) should also reduce the electric contact. In this sense, the current study is more realistic since the interface can exhibit imperfect elastic and electric bonding and becomes more representative of the principles of piezoelectricity.

However, to our knowledge, effective micro-macro methods for heterogeneous materials based on the twoscale asymptotic homogenization technique for piezoelectric composites with rhombic cells and mechanical/ electrical imperfect adhesion have not been reported completely. The novelty of this contribution is that the statement of the problem for piezoelectric composites with mechanical and electrical imperfect contact is given using the two-scale AHM. The associated local problems are derived and consequently use is made of potential methods of a complex variable and the properties of Weierstrass elliptic and related periodic functions to solve the problems on the rhombic unit cell. The complete set of elastic, piezoelectric and dielectric effective properties is obtained for a two-phase composite with electrical capacitors and mechanical springs between the fiber and the matrix as an idealization of electrical and mechanical imperfection conditions. Moreover, a three-phase model for piezoelectric composites containing perfect contact in the interfaces is proposed and a finite element method (FEM) approach is developed for composites with a spring-capacitor imperfect adherence.

\section{Formulation of the heterogeneous problem}

Consider piezoelectric materials that respond linearly to changes to mechanical and electrical fields. A twophase uniaxial reinforced composite is studied here, in which the fibers and the matrix have homogeneous and transversely isotropic properties. The axis of transverse 
symmetry coincides with the fiber direction, which is taken as the $\mathrm{Ox}_{3}$ axis. The fiber cross-section is circular. Moreover, the fibers are periodically distributed without overlapping in directions parallel to the $O w_{1}$ - and $O w_{2}$ axis, where $w_{1} \neq 0$ and $w_{2} \neq 0\left(w_{2} \neq \lambda w_{1}, \quad \lambda \in \mathbb{R}\right)$ are two complex numbers that define the rhombic periodic cell with an angle $\theta=\left(w_{1}, w_{2}\right)$. Therefore, the composite $\Omega\left(\equiv \Omega^{\varepsilon}\right)(\varepsilon$ is a small parameter defined below) consists of a rhombic array of identical circular fibers embedded in a homogeneous medium (Figure 1). The fibers are infinitely long.

The response of the material at the microscale level is analyzed using representative volume elements (RVEs) or basic cells. The fiber-reinforced material is assumed to have a periodic arrangement of fine scale fibers embedded in a matrix. A sample of a RVE is shown in Figure 1, where an appropriate periodic unit cell $S$ is taken as a regular rhomb in the $y_{1} y_{2}$-plane so that $S=S_{1} \cup S_{2}$ with $S_{1} \cap S_{2}=\emptyset$. The domain $S_{1}$ is occupied by the matrix and its complement $S_{2}$ by the fiber of radius R. A local Cartesian coordinate system $\mathbf{y}=\left(y_{1}, y_{2}, y_{3}\right)$ is introduced at the microscale and oriented in such a way that the $y_{3}$-axis is aligned parallel to the axis of the fibers. The microscale coordinates $\mathbf{y}$ of a point in the RVE are related to the macroscale coordinates $\mathbf{x}=\left(x_{1}, x_{2}, x_{3}\right)$ by $\mathbf{y}=\mathbf{x} / \varepsilon$, where $\varepsilon \ll 1$. Beside the use of subscripts, matrix and fiber-associated quantities are also referred to below by means of superscripts in brackets (1) and (2), respectively. A twophase composite is considered, which comprises a matrix with homogeneous properties given by the following property tensors: elastic $C_{i j k l}^{(1)}$, piezoelectric $e_{i j k}^{(1)}$ and dielectric permittivity $\kappa_{i j}^{(1)}$, in which parallel circular cylindrical fibers are embedded with corresponding homogeneous properties $C_{i j k l}^{(2)}, e_{i j k}^{(2)}$ and $\kappa_{i j}^{(2)}$.

In order to denote the dependence of a field variable on both the macroscale and the microscale coordinates, the superscript $\varepsilon$ is used, i.e. $\mathcal{F}^{\varepsilon}=\mathcal{F}(\mathbf{x}, \mathbf{y})$ where $\mathcal{F}^{\varepsilon}$ represents a scalar, vector or tensor field. All field variables are assumed to depend on the coordinates of both scales. For an arbitrary microstructure, material phases and therefore material properties such as elastic constants are functions of the microscale coordinates $\mathbf{y}$. In the following formulation an indicial and direct notation will be used interchangeably.

The coupled system of partial differential equations with rapidly oscillating coefficients for linear piezoelectric heterogeneous structures is

$$
\begin{aligned}
& \left(C_{i j k l}^{\varepsilon}(\mathbf{y}) u_{k, l}^{\varepsilon}+e_{k i j}^{\varepsilon}(\mathbf{y}) \phi_{, k}^{\varepsilon}\right)_{, j}=0, \\
& \left(e_{i k l}^{\varepsilon}(\mathbf{y}) u_{k, l}^{\varepsilon}-\kappa_{i k}^{\varepsilon}(\mathbf{y}) \phi_{, k}^{\varepsilon}\right)_{, i}=0 \text { in } \Omega
\end{aligned}
$$

Equation (1) represents a system of equations for finding $u_{i}$ and $\phi$ where $u_{k}^{\varepsilon}$ and $\phi^{\varepsilon}$ are the mechanical displacement and the electric potential, respectively. The material properties are piecewise functions. For simplicity, from now on the superscript $\varepsilon$ will be omitted on the material functions which satisfy the symmetries $\quad C_{i j k l}=C_{j i k l}=C_{k l i j}, \quad e_{k i j}=e_{k j i}, \kappa_{i k}=\kappa_{k i}$. In addition, the elasticity tensor and the dielectric permittivity tensor are assumed to be positive definite.

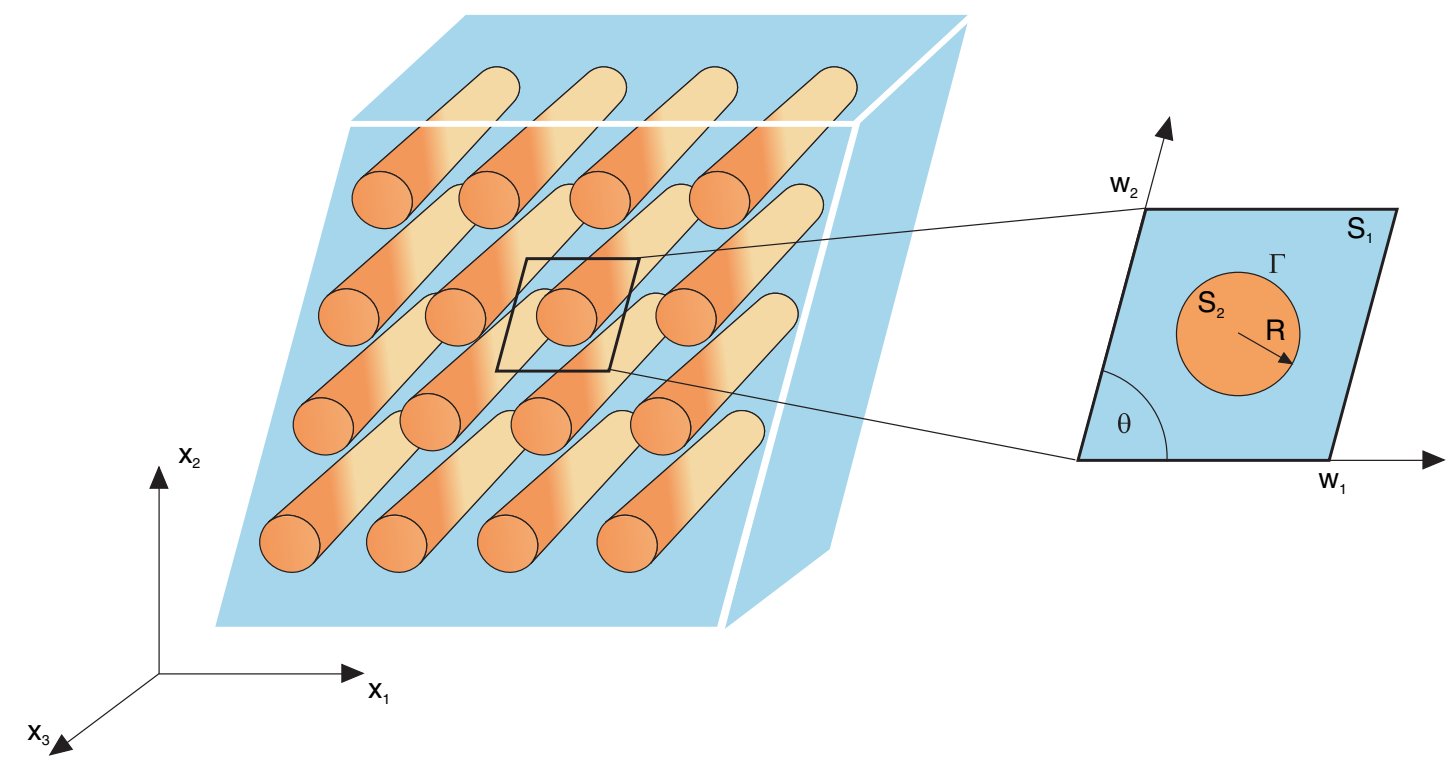

Figure I. Composite with rhombic array of circular cylinders embedded into a matrix and the unit cell. 
For a complete solution it is necessary to assign suitable boundary conditions, for instance

$$
u_{i}^{\varepsilon}=\hat{u}_{i} ; \quad \sigma_{i j}^{\varepsilon} n_{j}=\hat{S}_{i} ; \quad \phi^{\varepsilon}=\phi_{0} ; \quad D_{i}^{\varepsilon} n_{i}=0, \quad \text { on } \partial \Omega
$$

where $\hat{u}_{i}, \hat{S}_{i}$ and $\phi_{0}$ are the prescribed displacement, traction and electric potential on the boundary of the composite.

In the case of perfect bonding the continuities of displacement, traction, electric potential and normal electric displacement are enforced. In this section, an attempt is made to analyze the behavior of a piezocomposite under mechanical and electrical imperfect contacts.

\section{Spring-capacitor model and its mathematical formulation}

The electro-mechanical imperfect condition may be expressed as the simplest representation. The jumps in normal and tangential mechanical displacements are proportional to the associated traction components and the jumps of the electric potential are proportional to the electric displacement fields. ${ }^{11}$ Thus referring to

$$
\begin{gathered}
\llbracket \mathbf{T} \rrbracket=0, \quad \llbracket \mathbf{D} \rrbracket=0, \text { on } \Gamma \\
\mathbf{T}^{(\gamma)}=(-1)^{\gamma+1} \tilde{\mathbf{K}} \llbracket \mathbf{u} \rrbracket, \mathbf{D}^{(\gamma)} \cdot \mathbf{n}^{(\gamma)}=(-1)^{\gamma+1} \tilde{K}_{\phi} \llbracket \Phi \rrbracket, \text { on } \Gamma
\end{gathered}
$$

where the mechanical displacement, traction vectors and the spring stiffness matrix are written as

$$
\mathbf{u}=\left(\begin{array}{c}
u_{n} \\
u_{t} \\
u_{s}
\end{array}\right), \quad \mathbf{T}=\left(\begin{array}{c}
T_{n} \\
T_{t} \\
T_{s}
\end{array}\right), \quad \tilde{\mathbf{K}}=\left(\begin{array}{ccc}
\widetilde{K}_{n} & 0 & 0 \\
0 & \widetilde{K}_{t} & 0 \\
0 & 0 & \widetilde{K}_{s}
\end{array}\right)
$$

In these relations $\llbracket \bullet \rrbracket$ indicates the jump in the quantity - at the common interface $\Gamma\left(\equiv \Gamma^{\varepsilon}\right)$ between the fiber and the matrix; $\mathbf{n}$ is the outward unit normal vector on $\Gamma ; u_{n}, u_{t}, u_{s}$ are the normal and two tangential components of the mechanical displacement vector, respectively; $T_{n}, T_{t}, T_{s}$ are the normal and tangential components of the traction vector $\mathbf{T}$. The superscripts $\gamma, \gamma=1,2$ denote the matrix and fiber, respectively. The imperfect interface mechanical behavior would represent an idealization of a layer of three mechanical springs $\left(\tilde{K}_{n}\right.$ and $\left.\tilde{K}_{t}, \tilde{K}_{s}\right)$ with constants of zero thickness. The electrical imperfect interface is considered a distribution of electric capacitors of capacitance $\tilde{K}_{\phi}$ which is the capacitance of electric elements along the interface. The interface is a complete electric barrier when the capacitance is equal to zero. As the capacitance increases the level of electrical imperfection decreases, and perfect electrical interface prevails as the capacitance approaches infinity. The status of the electro-mechanical bonding is completely determined by appropriate values of these constants. For large enough values of the constants, the perfect bonding interface is recovered.

In order to study the imperfect contact condition, the relations between the displacement and traction vectors (5) are related to their Cartesian representations by the following expressions

$$
\begin{aligned}
& \left(\begin{array}{l}
u_{n} \\
u_{t} \\
u_{s}
\end{array}\right)=\left(\begin{array}{ccc}
\cos \varphi & \sin \varphi & 0 \\
-\sin \varphi & \cos \varphi & 0 \\
0 & 0 & 1
\end{array}\right)\left(\begin{array}{l}
u_{1} \\
u_{2} \\
u_{3}
\end{array}\right), \\
& \left(\begin{array}{l}
T_{n} \\
T_{t} \\
T_{s}
\end{array}\right)=\left(\begin{array}{ccc}
\cos \varphi & \sin \varphi & 0 \\
-\sin \varphi & \cos \varphi & 0 \\
0 & 0 & 1
\end{array}\right)\left(\begin{array}{l}
T_{1} \\
T_{2} \\
T_{3}
\end{array}\right)
\end{aligned}
$$

where the traction vector $\mathbf{T}\left(T_{i}=\sigma_{i j} n_{j}, \quad i, j=1,2,3\right)$ and $n_{j}$ (components of the outward unit normal vector) refer to the usual Cartesian coordinate system $\left(x_{1}, x_{2}, x_{3}\right)$.

Thus, the expression (3)-(4) on $\Gamma$ can be rewritten in the following form

$$
\begin{aligned}
\mathbf{T}^{(1)}+\mathbf{T}^{(2)} & =0, \quad \mathbf{D}^{(1)}+\mathbf{D}^{(2)}=0 \text { on } \Gamma, \\
T_{n}^{(\gamma)} & =(-1)^{\gamma+1} \widetilde{K}_{n} \llbracket u_{n} \rrbracket, T_{t}^{(\gamma)}=(-1)^{\gamma+1} \widetilde{K}_{t} \llbracket u_{t} \rrbracket, \\
T_{s}^{(\gamma)} & =(-1)^{\gamma+1} \widetilde{K}_{s} \llbracket u_{s} \rrbracket \text { on } \Gamma, \\
\mathbf{D}^{(\gamma)} \cdot \mathbf{n}^{(\gamma)} & =(-1)^{\gamma+1} \tilde{K}_{\phi} \llbracket \Phi \rrbracket \text { on } \Gamma
\end{aligned}
$$

\section{Two scales AHM and its consequences}

As discussed earlier, field variables are assumed to depend on both the macroscale coordinates $\mathbf{x}$ and microscale coordinates $\mathbf{y}=\mathbf{x} / \varepsilon$. Thus, the original problem (1), (2), (7) is studied via AHM. ${ }^{22}$ This method assumes a two-scale asymptotic expansion for the mechanical displacement and electric potential. ${ }^{18,23}$

Two sets of relevant functions can be derived which determinate the solution on the unit cell, i.e. the sets of $p q$-local functions, ${ }_{p q} \mathbf{M}(\mathbf{y})$ and ${ }_{p q} \mathbf{N}(\mathbf{y})$, and $p$-local functions, ${ }_{p} \mathbf{P}(\mathbf{y})$ and ${ }_{p} \mathbf{Q}(\mathbf{y})$, that depend only on $\mathbf{y}$. They are microscale characteristic functions, which relate the macroscale strain and electric field to the microscale fluctuations in the mechanical displacement and electric potential. They are the unique periodic solution of the so-called $p q$-local and $p$-local problems, denoted by ${ }_{p q} L$ and ${ }_{p} I$, respectively, over the periodic unit cell $S$, as it is defined below.

The ${ }_{p q} L$ problem seeks displacements ${ }_{p q} \mathbf{M}^{(\gamma)}(\mathbf{y})$ and potential ${ }_{p q} \mathbf{N}^{(\gamma)}(\mathbf{y})$ in $S_{\gamma}, \gamma=1,2$, which are periodic 
functions of periods $w_{1}=1, w_{2}=b e^{i \theta}, b>0$ is the modulus of this complex number and the local functions are the solution of the following equations

$$
\begin{aligned}
{ }_{p q} \sigma_{i \delta, \delta}^{(\gamma)} & =0 \text { in } S_{\gamma}, \\
{ }_{p q} D_{\delta, \delta}^{(\gamma)} & =0 \text { in } S_{\gamma}
\end{aligned}
$$

under mechanical and electrical imperfect conditions

$$
\begin{aligned}
& { }_{p q} \mathbf{T}^{(1)}{ }_{p q} \mathbf{T}^{(2)}=0, \llbracket{ }_{p q} \mathbf{D} \rrbracket=0 \quad \text { on } \quad \Gamma, \\
& { }_{p q} T_{n}^{(\gamma)}=(-1)^{\gamma+1} \widetilde{K}_{n} \llbracket{ }_{p q} M_{n} \rrbracket, \\
& { }_{p q} T_{t}^{(\gamma)}=(-1)^{\gamma+1} \widetilde{K}_{t} \llbracket p q \mathrm{M}_{t} \rrbracket, \\
& { }_{p q} T_{s}^{(\gamma)}=(-1)^{\gamma+1} \widetilde{K}_{s} \llbracket p q \mathbf{M}_{s} \rrbracket \text { on } \Gamma, \\
& { }_{p q} \mathbf{D}^{(\gamma)} \cdot \mathbf{n}^{(\gamma)}=(-1)^{\gamma+1} \tilde{K}_{\phi} \llbracket p q N \rrbracket \quad \text { on } \Gamma
\end{aligned}
$$

To assure the ${ }_{p q} L$ problems solution is unique, the functions also satisfy the condition that $\left.{ }_{p q} \mathbf{M}\right\rangle=\left\langle{ }_{p q} N\right\rangle=0$, where the angular brackets define the volume average per unit length over the periodic unit cell, that is, $\langle F\rangle=V^{-1} \int_{S} F(\mathbf{y}) \mathrm{d} \mathbf{y}$, where $\mathrm{V}$ is the periodic cell area.

Moreover,

$$
\begin{aligned}
{ }_{p q} \sigma_{i \delta}^{(\gamma)} & =C_{i \delta k \lambda}^{(\gamma)}{ }_{p q} M_{k, \lambda}^{(\gamma)}+e_{\lambda i \delta p q}^{(\gamma)} N_{, \lambda}^{(\gamma)}, \\
{ }_{p q} D_{\delta}^{(\gamma)} & =e_{\delta k \lambda p q}^{(\gamma)} M_{k, \lambda}^{(\gamma)}-\kappa_{\delta \lambda}^{(\gamma)}{ }_{p q} N_{, \lambda}^{(\gamma)}
\end{aligned}
$$

the comma notation denotes a partial derivative relative to the $y_{\delta}$ component, i.e. $U_{, \delta} \equiv \partial U / \partial y_{\delta}$; the summation convention is also understood for Greek indices, which run from 1 to 2; no summation is carried out over upper case indices, whether Latin or Greek. The functions ${ }_{p q} M_{t},{ }_{p q} M_{s},{ }_{p q} M_{n}$ are the two tangential and normal components of the vector ${ }_{p q} \mathbf{M}$ whereas ${ }_{p q} T_{t},{ }_{p q} T_{s},{ }_{p q} T_{n}$ are the two tangential and normal components of the traction vector ${ }_{p q} T_{i}={ }_{p q} \sigma_{i j}+$ $\left.C_{i j p q}\right) n_{j} \quad$ and $\quad{ }_{p q} D_{i}^{(\gamma)}=\left(C_{i j k l}^{(\gamma)} p q M_{k, l}^{(\gamma)}+e_{l p q}^{(\gamma)}{ }_{p q} N_{, l}^{(\gamma)}+\right.$ $\left.e_{i p q}^{(\gamma)}\right) n_{j}^{(\gamma)}$ associated to the local problem ${ }_{p q} L$. The expression of the traction vector can be written in terms of the tangential and normal components for each phase as $\left.{ }_{p q} T_{1}^{(\gamma)}=\left({ }_{p q} T_{n}^{(\gamma)}+C_{1 j p q}^{(\gamma)}\right) n_{j}^{(\gamma)},{ }_{p q} T_{2}^{(\gamma)}={ }_{p q} T_{t}^{(\gamma)}+C_{2 j p q}^{(\gamma)}\right) n_{j}^{(\gamma)}$, $\left.{ }_{p q} T_{3}^{(\gamma)}={ }_{p q} T_{s}^{(\gamma)}+C_{3 j p q}^{(\gamma)}\right) n_{j}^{(\gamma)}$. The symmetry between the indices $p$ and $q$ shows right away that at most six problems need to be considered at most.

Similarly, the ${ }_{p} I$ problem is stated as follows: the displacements ${ }_{p} \mathbf{P}^{(\gamma)}(\mathbf{y})$ and potential ${ }_{p} Q^{(\gamma)}(\mathbf{y})$ are sought in $S_{\gamma}, \gamma=1,2$, which are periodic functions with the same periods satisfying the following equations

$$
\begin{array}{ll}
{ }_{p} \sigma_{i \delta, \delta}^{(\gamma)}=0 & \text { in } S_{\gamma}, \\
{ }_{p} D_{\delta, \delta}^{(\gamma)}=0 & \text { in } S_{\gamma}
\end{array}
$$

and the above-mentioned interface conditions adapted to this problem, which are

$$
\begin{aligned}
& { }_{p} \mathbf{T}^{(1)}+{ }_{p} \mathbf{T}^{(2)}=0, \llbracket{ }_{p} \mathbf{D} \rrbracket=0 \text { on } \Gamma, \\
& { }_{p} T_{n}^{(\gamma)}=(-1)^{\gamma+1} \widetilde{K}_{n}\left[{ }_{p} P_{n} \rrbracket,\right. \\
& { }_{p} T_{t}^{(\gamma)}=(-1)^{\gamma+1} \widetilde{K}_{t} \llbracket{ }_{p} P_{t} \rrbracket, \\
& { }_{p} T_{s}^{(\gamma)}=(-1)^{\gamma+1} \widetilde{K}_{s} \llbracket{ }_{p} P_{s} \rrbracket \text { on } \Gamma, \\
& { }_{p} \mathbf{D}^{(\gamma)} \cdot \mathbf{n}^{(\gamma)}=(-1)^{\gamma+1} \tilde{K}_{\phi} \llbracket{ }_{p} Q \rrbracket \text { on } \Gamma
\end{aligned}
$$

where the functions ${ }_{p} P_{t},{ }_{p} P_{s},{ }_{p} P_{n}$ are the two tangential and normal components of the vector ${ }_{p} \mathbf{P}$ whereas ${ }_{p} T_{t},{ }_{p} T_{s},{ }_{p} T_{n}$, are the tangential and normal components of the traction vector ${ }_{p} T_{i}=\left({ }_{p} \sigma_{i j}+e_{p i j}\right) n_{j}$ and the electric field vector is written as ${ }_{p} D_{i}^{(\gamma)}=\left(e_{i k l}^{(\gamma)}{ }_{p} N_{k, l}^{(\gamma)}-\kappa_{l p}^{(\gamma)}{ }_{p} P_{, l}^{(\gamma)}+e_{i j p}^{(\gamma)}\right) n_{j}^{(\gamma)}$ associated to the local problem ${ }_{p} I$ and $\left\langle_{p} \mathbf{P}\right\rangle=\left\langle_{p} Q\right\rangle=0$, for the solution uniqueness. The traction vector can be rewritten in terms of the tangential and normal components for each phase as ${ }_{p} T_{1}^{(\gamma)}=\left({ }_{p} T_{n}^{(\gamma)}+e_{1 j p}^{(\gamma)}\right) n_{j}^{(\gamma)}$, ${ }_{p} T_{2}^{(\gamma)}=\left({ }_{p} T_{t}^{(\gamma)}+e_{2 j p}^{(\gamma)}\right) n_{j}^{(\gamma)},{ }_{p} T_{3}^{(\gamma)}=\left({ }_{p} T_{s}^{(\gamma)}+e_{3 j p}^{(\gamma)}\right) n_{j}^{(\gamma)}$.

Furthermore

$$
\begin{aligned}
{ }_{p} \sigma_{i \delta}^{(\gamma)} & =C_{i \delta k \lambda}^{(\gamma)} P_{k, \lambda}^{(\gamma)}+e_{\lambda i \delta p}^{(\gamma)} Q_{, \lambda}^{(\gamma)}, \\
{ }_{p} D_{\delta}^{(\gamma)} & =e_{\delta k \lambda}^{(\gamma)} P_{k, \lambda}^{(\gamma)}-\kappa_{\delta \lambda}^{(\gamma)}{ }_{p} Q_{, \lambda}^{(\gamma)}
\end{aligned}
$$

Also the non-homogeneous ${ }_{p} I$ problems contribute towards the homogenized properties.

The sets of the characteristic functions $p q$-functions ${ }_{p q} \mathbf{M}(\mathbf{y})$ and ${ }_{p q} \mathbf{N}(\mathbf{y})$, and $p$-functions ${ }_{p} \mathbf{P}(\mathbf{y})$ and ${ }_{p} \mathrm{Q}(\mathbf{y})$, must satisfy the following weak form equations ${ }^{18}$

$$
\begin{gathered}
\int_{Y}\left(C_{i j k l} \frac{\partial_{p q} M_{k}}{\partial y_{l}}-e_{k i j} \frac{\partial_{p q} N}{\partial y_{k}}\right) \frac{\partial v_{i}}{\partial y_{j}} \mathrm{~d} Y=\int_{Y} C_{i j p q} \frac{\partial v_{i}}{\partial y_{j}} \mathrm{~d} Y, \\
\int_{Y}\left(e_{k i j} \frac{\partial_{p} Q}{\partial y_{k}}-C_{i j k l} \frac{\partial_{p} P_{k}}{\partial y_{l}}\right) \frac{\partial v_{i}}{\partial y_{j}} \mathrm{~d} Y=\int_{Y} e_{q i j} \frac{\partial v_{i}}{\partial y_{j}} \mathrm{~d} Y, \\
\int_{Y}\left(e_{i k l} \frac{\partial_{p q} M_{k}}{\partial y_{l}}+\kappa_{i k} \frac{\partial_{p q} N}{\partial y_{k}}\right) \frac{\partial w}{\partial y_{i}} \mathrm{~d} Y=\int_{Y} e_{i p q} \frac{\partial w}{\partial y_{i}} \mathrm{~d} Y, \\
\int_{Y}\left(\kappa_{i k} \frac{\partial_{p} Q}{\partial y_{k}}+e_{i k l} \frac{\partial_{p} P_{k}}{\partial y_{l}}\right) \frac{\partial w}{\partial y_{i}} \mathrm{~d} Y=\int_{Y} \kappa_{i q} \frac{\partial w}{\partial y_{i}} \mathrm{~d} Y
\end{gathered}
$$

Equation (14) represents a system of partial differential equations that must be solved to obtain the microscale characteristic functions ${ }_{p q} \mathbf{M}(\mathbf{y}),{ }_{p q} \mathrm{~N}(\mathbf{y}),{ }_{p} \mathbf{P}(\mathbf{y})$ and ${ }_{p} \mathrm{Q}(\mathbf{y})$. The characteristic functions are subjected to periodic conditions over the boundary of $Y$. 
Table I. Material properties used in the calculations taken from Tita et al., ${ }^{21}$ Yan et al. ${ }^{30}$ and Pastor. ${ }^{31}$

\begin{tabular}{|c|c|c|c|c|c|c|}
\hline & Epoxy I & PZT-7A & Epoxy 2 & PZT-5 & Epoxy 3 & PZT-5A \\
\hline$C_{1 I}(\mathrm{GPa})$ & 8 & 148 & 4.445 & $133.9 \mid$ & 3.86 & 121.0 \\
\hline$C_{12}(\mathrm{GPa})$ & 4.4 & 76.2 & 2.189 & 68.98 & 2.57 & 75.4 \\
\hline$C_{13}(\mathrm{GPa})$ & 4.4 & 74.2 & 2.189 & 68.98 & 2.57 & 75.2 \\
\hline$C_{33}(\mathrm{GPa})$ & 8 & $13 \mid$ & 4.445 & $133.9 \mid$ & 3.86 & 111.0 \\
\hline$C_{44}(\mathrm{GPa})$ & 1.8 & 25.696 & 1.128 & 32.46 & 0.64 & 21.1 \\
\hline$C_{66}(\mathrm{GPa})$ & 1.8 & 35.9 & 1.128 & 32.46 & 0.64 & 22.8 \\
\hline$e_{31}\left(C / m^{2}\right)$ & - & -2.1 & - & -2.1 & - & -5.4 \\
\hline$e_{33}\left(C / m^{2}\right)$ & - & 12.3 & - & 9.5 & - & 15.8 \\
\hline$e_{15}\left(C / m^{2}\right)$ & - & 9.35 & - & 9.2 & - & 12.3 \\
\hline$\kappa_{11}(\mathrm{nC} / \mathrm{Vm})$ & 0.0372 & 4.065 & 0.079 & 4.07 I & 0.0797 & 8.11 \\
\hline$\kappa_{33}(\mathrm{nC} / \mathrm{Vm})$ & 0.0372 & 2.035 & 0.079 & 2.079 & 0.0797 & 7.35 \\
\hline
\end{tabular}

The linear piezoelectric theory constitutive relations for a heterogeneous and periodic medium $\Omega$ is characterized by the $Y$-periodic functions $\mathbf{C}(\mathbf{y}), \mathbf{e}(\mathbf{y}), \mathbf{\kappa}(\mathbf{y})$. The original constitutive relations with rapidly oscillating material coefficients are transformed into new physical relations with constant coefficients $\mathbf{C}^{*}, \mathbf{e}^{*}, \boldsymbol{\kappa}^{*}$ which represent the elastic, piezoelectric and permittivity properties, respectively, of an equivalent homogeneous medium and are called the composite effective coefficients of $\Omega$. Therefore, the system (1) can be transformed into an equivalent system with constant coefficients, which represents the overall properties of the composite.

The main problem to obtain such average formulae is to find the $S$-periodic solutions of nine ${ }_{p q} L,{ }_{p} I(p, q=1,2,3)$ local problems on $S$ in terms of the fast variable $\mathbf{y}$ based on the mathematical statement of both problems.

Once the local problems are solved, the homogenized properties $C_{i j p q}^{*}, e_{k i j}^{*}, \kappa_{i k}^{*}$ may be determined by using the following formulae

$$
\begin{aligned}
C_{i j p q}^{*} & =\left\langle C_{i j p q}+C_{i j k l} p q M_{k, l}+e_{k i j p q} N_{, k}\right\rangle, \\
e_{i p q}^{*} & =\left\langle e_{i p q}+e_{i k l ~ p q} M_{k, l}-\kappa_{i k p q} N_{, k}\right\rangle, \\
e_{p i j}^{*} & =\left\langle e_{p i j}+C_{i j k l} P_{k, l}+e_{k i j p} Q_{, k}\right\rangle, \\
\kappa_{i p}^{*} & =\left\langle\kappa_{i p}-e_{i k l p} P_{k, l}+\kappa_{i k p} Q_{, k}\right\rangle
\end{aligned}
$$

Each local problem (8)-(9) and (11)-(12) ( $p, q=1,2,3)$ uncouples into two sets of equations for finding the local functions ${ }_{p q} \mathbf{M}(\mathbf{y}),{ }_{p q} \mathbf{N}(\mathbf{y})$, and ${ }_{p} \mathbf{P}(\mathbf{y}),{ }_{p} \mathbf{Q}(\mathbf{y})$. The plane and antiplane-strain systems of equations correspond to five plane-strain local problems ${ }_{p p} L,{ }_{12} L,{ }_{3} I$ and four antiplane-strain ones ${ }_{13} L,{ }_{23} L,{ }_{1} I,{ }_{2} I$. The effective coefficients involved in each local problem is given in Table 1 of the recent report. ${ }^{17}$
The piezoelectric composite global behavior is related to the monoclinic 2 class symmetry which contains 13 elastic, 8 piezoelectric and 4 dielectric independent coefficients. ${ }^{24}$

A detailed description of the local-value problems solution set up in (8)-(9), (11)-(12) using complex variable methods, the properties of doubly periodic elliptic and related functions with periods $w_{1}$ and $w_{2}$ applied to such problems has not been reported until now. The local-value problems solution under mechanical imperfect and electrical perfect conditions is reported in Rodriguez-Ramos et al. ${ }^{17}$ Taking into account that the rate of debonding depends on material property and interphase thickness, the following relations are used $\tilde{K}_{n}=C_{11}^{(I)} / t, \quad \tilde{K}_{t}=C_{44}^{(I)} / t, \tilde{K}_{s}=C_{44}^{(I)} / t, \tilde{K}_{\phi}=\kappa_{11}^{(I)} / t$ assuming the isotropic and thus non-piezoelectric behavior of interphase material.

Since the electrical imperfection is related only with the antiplane problem we focus our aim in the out-ofplane problems where the mechanical and electrical imperfect conditions are coupled. For convenience we use in all subsequent derivations dimensionless imperfection parameters $K_{s}$ and $K_{\phi}$ which are related to $\tilde{K}_{s}$ and $\tilde{K}_{\phi}$ by $K_{s}=\tilde{K}_{s} t / C_{44}^{(I)}$ and $K_{\phi}=\tilde{K}_{\phi} t / \kappa_{11}^{(I)}$.

The thickness interphase is denoted by $t$. The originality of this work is the study of antiplane problems since the mechanical and electrical imperfect conditions are coupled. In this case, for the spring-capacitor model, the only imperfect parameters involved are $K_{t}, K_{s}$ for mechanical contribution and $K_{\varphi}$ for the electrical one.

\section{Antiplane problems solution}

Now, the problem ${ }_{13} L$ is considered in detail from the set of antiplane problems ${ }_{13} L,{ }_{23} L,{ }_{1} I,{ }_{2} I$. From now on, the preindices are not used and the effective properties 
are denoted with the Voigt two indices short notation. The shear piezoelectric effective properties determination denoted by $C_{44}^{*}, C_{45}^{*}, C_{55}^{*}$ (shear moduli), $e_{15}^{*}, e_{14}^{*}, e_{24}^{*}$ (shear stress piezoelectric coefficients) and $\kappa_{11}^{*}, \kappa_{12}^{*}, \kappa_{22}^{*}$ (transverse permittivity constants) is the main aim of this part where the constituents of each phase of the composite are of the crystal class symmetry $6 \mathrm{~mm}$. In this case, the relevant constitutive relations are

$$
\begin{aligned}
& \sigma_{23}=2 C_{44} \varepsilon_{23}-e_{15} E_{2}, \quad \sigma_{13}=2 C_{44} \varepsilon_{13}-e_{15} E_{1}, \\
& D_{1}=2 e_{15} \varepsilon_{23}+\kappa_{11} E_{1}, \quad D_{2}=2 e_{15} \varepsilon_{13}+\kappa_{11} E_{2}
\end{aligned}
$$

The displacement $M \equiv{ }_{13} M_{s}$ and potential $N \equiv{ }_{13} N_{s}(s$ denotes the aforementioned tangential component), which appear in (15) are the unique solution of the above-mentioned local problem ${ }_{13} L$. Therefore, equation (8) yields

$$
\Delta M^{(\gamma)}=0, \Delta N^{(\gamma)}=0 \text { in } Y_{\gamma}
$$

where $\Delta$ is the two-dimensional Laplacian and the contact conditions (9) on $\Gamma$ are written in the form

$$
\begin{aligned}
T_{s}^{(1)}+T_{s}^{(2)} & =0, \mathrm{D}_{\delta}^{(1)}+\mathrm{D}_{\delta}^{(2)}=0 \text { on } \Gamma, \\
\left(C_{44}^{(\gamma)} M_{, \delta}^{(\gamma)}+e_{15}^{(\gamma)} N_{, \delta}^{(\gamma)}\right) n_{\delta}+C_{44}^{(\gamma)} n_{1} & =(-1)^{\gamma+1} K_{s} C_{44}^{(1)} \llbracket M \rrbracket R^{-1} \text { on } \Gamma, \\
D_{\delta}^{(\gamma)} . n_{\delta}^{(\gamma)} & =(-1)^{\gamma+1} K_{\phi} \kappa_{11}^{(1)} \llbracket N \rrbracket \text { on } \Gamma
\end{aligned}
$$

Equation (15) are transformed to area integrals applying Green's theorem. The doubly periodic boundary conditions on $Y$ and the continuity of displacement and potential on $\Gamma$ leads to

$$
\begin{aligned}
C_{55}^{*}-i C_{45}^{*}= & \left\langle C_{55}\right\rangle+(-1)^{\gamma} \frac{C_{55}^{(\gamma)}}{V} \int_{\Gamma} M^{(\gamma)} \mathrm{d} y_{2}+i M^{(\gamma)} \mathrm{d} y_{1} \\
& +(-1)^{\gamma} \frac{e_{15}^{(\gamma)}}{V} \int_{\Gamma} N^{(\gamma)} \mathrm{d} y_{2}+i N^{(\gamma)} \mathrm{d} y_{1}, \\
e_{15}^{*}-i e_{14}^{*}= & \left\langle e_{15}\right\rangle+(-1)^{\gamma} \frac{e_{15}^{(\gamma)}}{V} \int_{\Gamma} M^{(\gamma)} \mathrm{d} y_{2}+i M^{(\gamma)} \mathrm{d} y_{1} \\
& +(-1)^{\gamma} \frac{\kappa_{11}^{(\gamma)}}{V} \int_{\Gamma} N^{(\gamma)} \mathrm{d} y_{2}+i N^{(\gamma)} \mathrm{d} y_{1}
\end{aligned}
$$

where summation convention is understood for $\gamma$, which run from 1 to 2 .

Methods of potential theory are used to solve (18). Doubly periodic harmonic functions are to be found in terms of the following harmonic functions Laurent and Taylor expansions:

$$
M^{(1)}(z)=\operatorname{Re}\left\{\frac{z}{R} a_{0}+\sum_{p=1}^{\infty} o\left(\frac{R}{z}\right)^{p} a_{p}+\sum_{k=1}^{\infty}{ }^{o} \sum_{p=1}^{\infty} o\left(\frac{z}{R}\right)^{p} \eta_{k p} a_{k}\right\},
$$

$$
\begin{aligned}
& N^{(1)}(z)=\operatorname{Re}\left\{\frac{z}{R} b_{0}+\sum_{p=1}^{\infty} o\left(\frac{R}{z}\right)^{p} b_{p}+\sum_{k=1}^{\infty} o \sum_{p=1}^{\infty} o\left(\frac{z}{R}\right)^{p} \eta_{k p} b_{k}\right\} \text { in } S_{1} \\
& M^{(2)}(z)=\operatorname{Re}\left\{\sum_{p=1}^{\infty}{ }^{o} c_{p}\left(\frac{z}{R}\right)^{p}\right\}, \quad N^{(2)}(z)=\operatorname{Re}\left\{\sum_{p=1}^{\infty}{ }^{o} d_{p}\left(\frac{z}{R}\right)^{p}\right\} \text { in } S_{2}
\end{aligned}
$$

where $\quad \eta_{k l}=-\frac{(k+l-1) !}{(k-1) ! l !} R^{k+l} \sum_{m=-\infty}^{\infty} \sum_{n=-\infty}^{\infty} \frac{1}{\left(m w_{1}+n w_{2}\right)^{k+l}}$, $m+n \neq 0, \quad k+l>2$ and $a_{p}, b_{p}, c_{p}, d_{p}$ are complex undetermined coefficients; $w_{1}, w_{2}$ are the rhombic array periods, respectively (see Figure 1). The superscript "o" next to the summation symbol means that " $p$ " runs only over odd integers so that each term in (20) has the same anti-symmetry property as $M^{(\gamma)}$ and $N^{(\gamma)}$, namely, $M^{(\gamma)}(-z)=-M^{(\gamma)}(z), \quad N^{(\gamma)}(-z)=$ $-N^{(\gamma)}(z){ }^{23,25}$

The line integrals in (19) and the assumed expansions (20) lead to a very simple result because of the trigonometric functions orthogonality, namely

$$
\begin{aligned}
& \left.\int_{\Gamma} M^{(1)} \mathrm{d} x_{2}+i M^{(1)} \mathrm{d} x_{1}=\pi R \quad \bar{a}_{1}+a_{0}+\sum_{k=1}^{\infty}{ }^{o} \eta_{k 1} a_{k}\right), \\
& \left.\int_{\Gamma} N^{(1)} \mathrm{d} x_{2}+i N^{(1)} \mathrm{d} x_{1}=\pi R \quad \bar{b}_{1}+b_{0}+\sum_{k=1}^{\infty}{ }^{o} \eta_{k 1} b_{k}\right), \\
& \int_{\Gamma} M^{(2)} \mathrm{d} x_{2}+i M^{(2)} \mathrm{d} x_{1}=\pi R c_{1}, \\
& \int_{\Gamma} N^{(2)} \mathrm{d} x_{2}+i N^{(2)} \mathrm{d} x_{1}=\pi R d_{1}
\end{aligned}
$$

Substituting (21) into equation (19), and taking into consideration the imperfect contact condition (18), we obtain the final expression of the effective coefficients

$$
\begin{aligned}
C_{55}^{*}-i C_{45}^{*} & =C_{55}^{(1)}\left(1-2 V_{2} \Pi_{11}\right), \\
e_{15}^{*}-i e_{14}^{*} & =\sqrt{C_{55}^{(1)} \kappa_{11}^{(1)}}\left(E_{15}^{(1)}-2 V_{2} \Pi_{21}\right)
\end{aligned}
$$

where

$$
\begin{aligned}
& \Pi_{11}=\bar{a}_{1}+E_{15}^{(1)} \bar{b}_{1}, \quad \Pi_{21}=E_{15}^{(1)} \bar{a}_{1}-\bar{b}_{1}, \\
& E_{15}^{(\gamma)}=e_{15}^{(\gamma)} / \sqrt{C_{55}^{(\gamma)} \kappa_{11}^{(\gamma)}},
\end{aligned}
$$


the overbar denotes complex conjugate numbers, the fiber volume fraction is $V_{2}=\pi R^{2} / V$, $V=\left|w_{1}\right|\left|w_{2}\right| \sin \theta$ denotes the area of periodic cell. The unknown constants $a_{1}, b_{1}$ are found from the solutions of two infinite systems related to the local problems ${ }_{13} L$, in which only the residue of $M^{(\gamma)}$ and $N^{(\gamma)}$ contribute towards $C_{55}^{*}, C_{45}^{*}$ and $e_{15}^{*}, e_{14}^{*}$. Thus, expressions for $a_{1}, b_{1}$ are now sought from the system of infinite equations

$$
\mathcal{M} \times \mathcal{D}=\mathcal{U}
$$

where the vector $\mathcal{D}^{T}=\left(x_{1}, x_{2}, x_{3}, x_{4}\right)$ contains the real and imaginary parts of the unknown $a_{1}=x_{1}+i x_{2}, \quad b_{1}=x_{3}+i x_{4}$ and the inhomogeneous vector $\mathcal{U}$ is given by $\mathcal{U}^{T}=R\left(\delta_{1 \alpha} \lambda_{1}, \delta_{2 \alpha} \lambda_{1}, \delta_{1 \alpha} \lambda_{2}, \delta_{2 \alpha} \lambda_{2}\right)$. The superindex $T$ denotes transpose and the $4 \times 4$ order matrix $\mathcal{M}\left(m_{n k}\right)$ is defined by the following matrix form

$$
\begin{aligned}
& \mathcal{M}=\mathcal{K}+R^{2} \mathcal{J}-\mathcal{N}_{1} \mathcal{P}^{-1} \mathcal{N}_{2} \\
& \begin{array}{c}
h_{12}=\Re e\left\{\frac{\delta_{1} \bar{w}_{2}-\delta_{2} \bar{w}_{1}}{w_{1} \bar{w}_{2}-w_{2} \bar{w}_{1}}\right\}, \quad h_{21}=\Im m\left\{\frac{\bar{\delta}_{1} \bar{w}_{2}-\bar{\delta}_{2} \bar{w}_{1}}{w_{1} \bar{w}_{2}-w_{2} \bar{w}_{1}}\right\}, \\
h_{22}=\Im m\left\{\frac{\delta_{1} \bar{w}_{2}-\delta_{2} \bar{w}_{1}}{w_{1} \bar{w}_{2}-w_{2} \bar{w}_{1}}\right\}, \quad \delta_{\gamma}=2 \zeta\left(w_{\gamma} / 2\right),
\end{array} \\
& \text { 促 }
\end{aligned}
$$

$\zeta(z)$ is the Zeta quasi periodic Weierstrass function defined as $\zeta(z)=\frac{1}{z}+\sum_{m, n}^{\infty}{ }^{\prime}\left(\frac{1}{z-T_{m n}}+\frac{1}{T_{m n}}+\frac{z}{T_{m n}^{2}}\right)$, $T_{m n}=m w_{1}+n w_{2}$ and the prime over the summation symbol means that the pair $(m, n)=(0,0)$ is excluded. The Legendre's relationship links $\delta_{1}, \delta_{2}$ and the periods $w_{1}, w_{2}: \delta_{1} w_{2}-\delta_{2} w_{1}=\pi i$. The Laurent series expansion of $\zeta$ is $\zeta(z)=\frac{1}{z}-\sum_{k=2}^{\infty} c_{k} \frac{z^{2 k-1}}{2 k-1}$, where $c_{1}=0, c_{2}=3 S_{4}, c_{3}=5 S_{6} \quad$ and $c_{k}=\frac{3}{(2 k+1)(k-3)} \sum_{m=2}^{k-2} c_{m} c_{k-m}, \quad k \geq 4$. The lattice $S_{k}$ is defined by $S_{k}=\sum_{m, n}\left(m w_{1}+n w_{2}\right)^{-k}, \quad m^{2}+n^{2} \neq 0$, $k>2, S_{2}=0$. In particular only $S_{4}$ and $S_{6}$ are used in the numerical implementation; these are given in Table 1 of Chih-Bing ${ }^{26}$ for parallelogramic and rhombic cells, respectively.

The matrices $\mathcal{N}_{1}, \mathcal{P}$ and $N_{2}$ are of infinite order. For the numerical implementation it is necessary to truncate to certain order $n \in \mathbb{N}$. The matrix

$$
\begin{aligned}
\mathcal{K}= & \left(\begin{array}{cccc}
\beta_{11} & 0 & \alpha_{11} & 0 \\
0 & \beta_{11} & 0 & \alpha_{11} \\
\beta_{31} & 0 & \alpha_{31} & 0 \\
0 & \beta_{31} & 0 & \alpha_{31}
\end{array}\right), \quad \mathcal{J}=\left(\begin{array}{ccc}
\beta_{21}\left(\begin{array}{cc}
h_{11}+h_{12} & h_{21}-h_{22} \\
-\left(h_{21}+h_{22}\right) & h_{11}-h_{12}
\end{array}\right) & \alpha_{21}\left(\begin{array}{cc}
h_{11}+h_{12} & h_{21}-h_{22} \\
-\left(h_{21}+h_{22}\right) & h_{11}-h_{12}
\end{array}\right) \\
\beta_{41}\left(\begin{array}{ccc}
h_{11}+h_{12} & h_{21}-h_{22} \\
-\left(h_{21}+h_{22}\right) & h_{11}-h_{12}
\end{array}\right) & \alpha_{41}\left(\begin{array}{cc}
h_{11}+h_{12} & h_{21}-h_{22} \\
-\left(h_{21}+h_{22}\right) & h_{11}-h_{12}
\end{array}\right)
\end{array}\right) \\
& \text { with } h_{11}=\Re e\left\{\frac{\bar{\delta}_{1} \bar{w}_{2}-\bar{\delta}_{2} \bar{w}_{1}}{w_{1} \bar{w}_{2}-w_{2} \bar{w}_{1}}\right\}
\end{aligned}
$$

is composed of submatrices $\left(\mathcal{P}_{\mathrm{ts}}\right)_{4 \times 4}$, defined by

$$
\mathcal{P}=\left(\begin{array}{ccc}
\mathcal{P}_{11} & \ldots & \mathcal{P}_{1 \mathrm{n}} \\
\vdots & \ldots & \vdots \\
\mathcal{P}_{\mathrm{n} 1} & \ldots & \mathcal{P}_{n n}
\end{array}\right)_{4 \mathrm{n} \times 4 \mathrm{n}}
$$

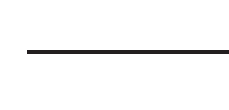

$$
\begin{aligned}
& \mathcal{K}=\left(\begin{array}{cccc}
\beta_{12 t+1} & 0 & \alpha_{12 t+1} & 0 \\
0 & \beta_{12 t+1} & 0 & \alpha_{12 t+1} \\
\beta_{32 t+1} & 0 & \alpha_{32 t+1} & 0 \\
0 & \beta_{32 t+1} & 0 & \alpha_{32 t+1}
\end{array}\right) \\
& Z_{t s}=\left(\begin{array}{ll}
\beta_{22 t+1}\left(\begin{array}{lll}
w_{12 t+1} 2 s+1 & -w_{22 t+1} 2 s+1 \\
-w_{22 t+1} 2 s+1 & -w_{12 t+1} 2 s+1
\end{array}\right) & \alpha_{22 t+1}\left(\begin{array}{ccc}
w_{12 t+1} 2 s+1 & -w_{22 t+1} 2 s+1 \\
-w_{22 t+1} 2 s+1 & -w_{12 t+1} 2 s+1
\end{array}\right) \\
\beta_{42 t+1}\left(\begin{array}{lll}
w_{12 t+1} 2 s+1 & -w_{22 t+1} 2 s+1 \\
-w_{22 t+12 s+1} & -w_{12 t+1} 2 s+1
\end{array}\right) & \alpha_{42 t+1}\left(\begin{array}{ccc}
w_{12 t+1} 2 s+1 & -w_{2} 2 t+12 s+1 \\
-w_{2} 2 t+12 s+1 & -w_{12 t+1} 2 s+1
\end{array}\right)
\end{array}\right)
\end{aligned}
$$
$\mathcal{P}_{\mathrm{ts}}=\delta_{\mathrm{ts}} \mathcal{K}+\mathcal{Z}_{\mathrm{ts}}$ 
$w_{1 k p}=\Re e\left(w_{k p}\right), \quad w_{2 k p}=\Im m\left(w_{k p}\right)$ are the complex number real and imaginary parts $w_{k p}=\frac{(k+p-1) !}{(k-1) !(p-1) !}$ $\frac{R^{k+p}}{\sqrt{k p}} S_{k+p}, \quad k=2 t-1, p=2 s-1, \quad t, s=1,2,3 \ldots,$. The matrices $\mathcal{N}_{1}=\left(n_{41} \cdots n_{4 \mathrm{n}}\right)_{4 \times 4 n} \quad$ and $\mathcal{N}_{2}=\left(\begin{array}{c}n_{14} \\ \vdots \\ n_{n 4}\end{array}\right)_{4 n \times 4}$ are composed by sub-matrices $\left(n_{4 t}\right)_{4 \times 4}$ and $\left(n_{t 4}\right)_{4 \times 4}$ defined by $n_{4 \mathrm{k}}=Z_{2 \mathrm{t}+1} 1$, $n_{\mathrm{t} 4}=Z_{12 \mathrm{t}+1}$, respectively. The magnitudes $\beta_{1 \mathrm{p}}, \beta_{2 \mathrm{p}}, \beta_{3 \mathrm{p}}, \beta_{4 \mathrm{p}}, \alpha_{1 \mathrm{p}}, \alpha_{2 \mathrm{p}}, \alpha_{3 \mathrm{p}}, \alpha_{4 \mathrm{p}}$ are given as follows,
As a summary, all the effective coefficients derived from the local problems can be listed as follows

$$
\begin{aligned}
& C_{55}^{*}-i C_{45}^{*}=C_{55}^{(1)}\left(1-2 V_{2} H_{11}\right), \\
& C_{45}^{*}-i C_{44}^{*}=-C_{55}^{(1)}\left(i+2 V_{2} H_{12}\right), \\
& e_{15}^{*}-i e_{14}^{*}=\sqrt{C_{55}^{(1)} \kappa_{11}^{(1)}}\left(E_{15}^{(1)}-2 V_{2} H_{21}\right), \\
& e_{14}^{*}-i e_{24}^{*}=-\sqrt{C_{55}^{(1)} \kappa_{11}^{(1)}}\left(i E_{15}^{(1)}+2 V_{2} H_{22}\right),
\end{aligned}
$$

$$
\begin{array}{lc}
\beta_{1 p}=1, & \beta_{3 p}=1, \\
\beta_{2 p}=\frac{1-\chi_{p}\left(1-p / K_{s}\right)-\sqrt{\chi_{p} \chi_{t}} E_{15}^{(1)} E_{15}^{(2)} p / K_{\phi}}{1+\chi_{p}\left(1+p / K_{s}\right)-\sqrt{\chi_{p} \chi_{t}} E_{15}^{(1)} E_{15}^{(2)} p / K_{\phi}}, & \beta_{4 p}=\frac{E_{15}^{(1)}-\sqrt{\chi_{p} \chi_{t}} E_{15}^{(2)}\left(1-p / K_{s}\right)+\chi_{t} E_{15}^{(1)} p / K_{\phi}}{E_{15}^{(1)}+\sqrt{\chi_{p} \chi_{t}} E_{15}^{(2)}\left(1+p / K_{s}\right)+\chi_{t} E_{15}^{(1)} p / K_{\phi}}, \\
\alpha_{1 p}=\frac{E_{15}^{(1)}+\chi_{p} E_{15}^{(1)} p / K_{s}+\sqrt{\chi_{p} \chi_{t}} E_{15}^{(2)}\left(1+p / K_{\phi}\right)}{1+\chi_{p}\left(1+p / K_{s}\right)-\sqrt{\chi_{p} \chi_{t}} E_{15}^{(1)} E_{15}^{(2)} p / K_{\phi}}, & \alpha_{3 p}=\frac{-1+\sqrt{\chi_{p} \chi_{t}} E_{15}^{(1)} E_{15}^{(2)} p / K_{s}-\chi_{t}\left(1+p / K_{\phi}\right)}{E_{15}^{(1)}+\sqrt{\chi_{p} \chi_{t}} E_{15}^{(2)}\left(1+p / K_{s}\right)+\chi_{t} E_{15}^{(1)} p / K_{\phi}}, \\
\alpha_{2 p}=\frac{E_{15}^{(1)}+\chi_{p} E_{15}^{(1)} p / K_{s}-\sqrt{\chi_{p} \chi_{t}} E_{15}^{(2)}\left(1-p / K_{\phi}\right)}{1+\chi_{p}\left(1+p / K_{s}\right)-\sqrt{\chi_{p} \chi_{t}} E_{15}^{(1)} E_{15}^{(2)} p / K_{\phi}}, & \alpha_{4 p}=\frac{-1+\sqrt{\chi_{p} \chi_{t}} E_{15}^{(1)} E_{15}^{(2)} p / K_{s}+\chi_{t}\left(1-p / K_{\phi}\right)}{E_{15}^{(1)}+\sqrt{\chi_{p} \chi_{t}} E_{15}^{(2)}\left(1+p / K_{s}\right)+\chi_{t} E_{15}^{(1)} p / K_{\phi}}
\end{array}
$$

where $\chi_{p}=C_{44}^{(2)} / C_{44}^{(1)}, \chi_{t}=\kappa_{11}^{(2)} / \kappa_{11}^{(1)}$. The inhomogeneous terms $\left(\delta_{1 \alpha} \lambda_{1}, \delta_{2 \alpha} \lambda_{1}, \delta_{1 \alpha} \lambda_{2}, \delta_{2 \alpha} \lambda_{2}\right)$ for the local problems ${ }_{\alpha 3} L$ are $\lambda_{1}=\beta_{21}, \lambda_{2}=\beta_{41}$ and for the local problems ${ }_{\alpha} L\left({ }_{1} L\right.$ and $\left.{ }_{2} L\right)$, are

$$
\begin{aligned}
& \lambda_{1}=\frac{E_{15}^{(1)}-\sqrt{\chi_{p} \chi_{t}} E_{15}^{(2)}+\chi_{p} E_{15}^{(1)} / K_{s}+\sqrt{\chi_{p} \chi_{t}} E_{15}^{(2)} / K_{\phi}}{1+\chi_{p}\left(1+K_{s}^{-1}\right)-\sqrt{\chi_{p} \chi_{t}} E_{15}^{(1)} E_{15}^{(2)} / K_{\phi}}, \\
& \lambda_{2}=\frac{-1+\chi_{t}+\sqrt{\chi_{p} \chi_{t}} E_{15}^{(1)} E_{15}^{(2)} / K_{s}-\chi_{t} / K_{\phi}}{E_{15}^{(1)}+\sqrt{\chi_{p} \chi_{t}} E_{15}^{(2)}\left(1+K_{s}^{-1}\right)+\chi_{t} E_{15}^{(1)} / K_{\phi}} .
\end{aligned}
$$

The limit case of perfect contact condition for piezoelectric antiplane problem is the result of a particular case of (23)-(25) as $K_{s} \rightarrow \infty, \quad K_{\phi} \rightarrow \infty$ and the same results reported ${ }^{23,25}$ are then reproduced. In this case, the parameters $a_{1}, b_{1}$ are the same that formula (3.25) page 1475 reported by Sabina et al. ${ }^{25}$ The infinite system (23)-(25) is used such that it is truncated too obtaining an $n \times n$ order system. It is interesting to note that the effective properties are monotonic functions of order $n$ of the solution of the system. The numerical results converge well to the exact solutions when an adequate order in the solution of the system is chosen as $n$ increases. The truncation order for solving the system increases as the parameters $K_{s}, K_{\phi} \chi^{*}$ and the fiber volume fraction are high. In the numerical examples the solutions are given for $N=10$, because this order of $N$ achieves the required accuracy for the used parameters.

$$
\begin{gathered}
\kappa_{11}^{*}-i \kappa_{12}^{*}=\kappa_{11}^{(1)}\left(1+2 V_{2} H_{31}\right), \\
\kappa_{12}^{*}-i \kappa_{22}^{*}=-\kappa_{11}^{(1)}\left(i-2 V_{2} H_{31}\right)
\end{gathered}
$$

where $H_{1 \alpha}={ }_{\alpha 3} \bar{a}_{1}+{ }_{\alpha 3} \bar{b}_{1} E, H_{2 \alpha}={ }_{\alpha 3} \bar{a}_{1} E-{ }_{\alpha 3} \bar{b}_{1}, H_{3 \alpha}=$ ${ }_{\alpha} \bar{a}_{1} E-{ }_{\alpha} \bar{b}_{1}$, and ${ }_{\alpha 3} a_{1},{ }_{\alpha 3} b_{1},{ }_{\alpha} a_{1}$ and ${ }_{\alpha} b_{1}$ are the infinite systems solution related to the local problems ${ }_{13} L,{ }_{23} L$, ${ }_{1} I$ and ${ }_{2} I$.

\section{Three-phase piezoelectric composite}

Now, we consider a three-phase composite. The interface conditions between the two contiguous phases, occupied by $S_{\gamma}$, are assumed to be in perfect contact along the interfaces $\Gamma_{\gamma}, \gamma=1,2$ of each cylinder. The displacement and quasi-static electric potentials, traction and normal electric displacement are continuous across the interfaces $\Gamma_{\gamma}$, between the phases (Figure 2). Therefore, the perfect contact condition can be written as

$$
\begin{gathered}
\left\|u_{i}\right\|_{\gamma}=0, \quad\|\phi\|_{\gamma}=0 \\
\left\|\sigma_{i j} n_{j}\right\|_{\gamma}=0, \quad\left\|D_{i} n_{i}\right\|_{\gamma}=0
\end{gathered}
$$

the double bar notation $\|f\|_{\gamma}$ is used to denote the jump of facross the interphase $\Gamma_{\gamma}$, i.e. $\|f\|_{1}=f^{(1)}-f^{(I)}$ and $\|f\|_{2}=f^{(I)}-f^{(2)}$, whereas the indices (1), (I) and (2) denote matrix, interphase and fiber properties, 


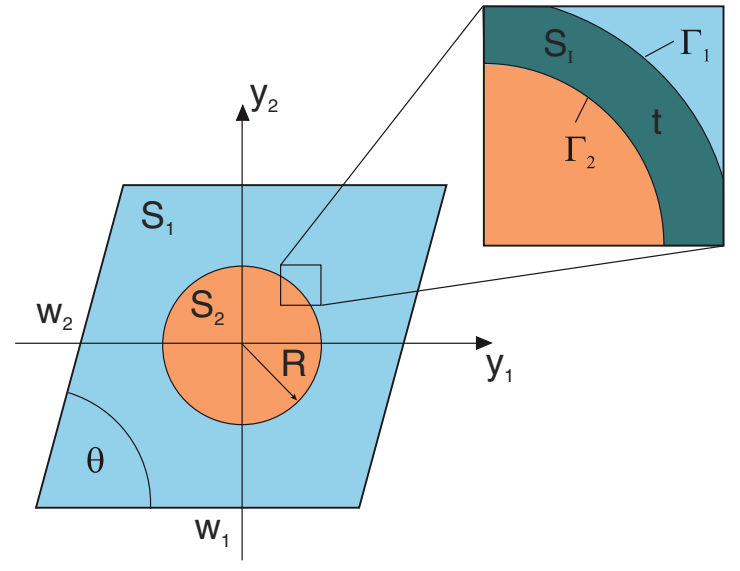

Figure 2. Three-phase composite under study.

respectively. Besides, $n_{i}$ is the outward unit normal vector component $\mathbf{n}$ to the interface $\Gamma_{\gamma}$.

\section{Method of solution}

By means of the AHM, ${ }^{27}$ it is possible to obtain from (1)-(2) an asymptotic solution of the aforementioned problem, the local problems on the periodic cell and the effective coefficients, analogous to those reported in Guinovart-Díaz et al., ${ }^{28}$ being the main goal of this work. It is a well-known derivation whose details can be found elsewhere ${ }^{13-15}$ and it is omitted here.

Of a greater interest are the so-called local (or canonical) problems associated here to the local cell problems.

Nine local problems arise, ${ }_{\alpha q} L$ and ${ }_{\alpha} I$ $(\alpha=1,2 ; q=1,2,3)$, over the periodic unit cell $S$ can be determined. Only four local problems out of these nine are defined as antiplane problems, which are referred also as ${ }_{13} L,{ }_{23} L,{ }_{1} I$ and ${ }_{2} I$. The remaining problems are of the plane strain kind.

\section{Antiplane local problems ${ }_{\alpha 3} L,(\alpha=1,2)$}

The mathematical statement of local problems ${ }_{\alpha 3} L$ is formulated. For simplicity, only the main results of the local problem ${ }_{\alpha 3} L$ are shown and the pre-index will be omitted in the expressions in this section. The fundamental problem consists in finding the functions $M^{(s)}$ and $N^{(s)}(s=1, I, 2)$ that satisfies Laplace equations, the perfect conditions at the interfaces $\Gamma_{\gamma}$ and the null average over the periodic cell

$$
\begin{aligned}
& \left(C_{i j 3 k}^{(s)} M_{, k}^{(s)}+e_{l i j}^{(s)} N_{, k}^{(s)}\right)_{, j}=0, \\
& \left(e_{i 3 k}^{(s)} M_{, k}^{(s)}-\kappa_{i k}^{(s)} N_{, k}^{(s)}\right)_{, j}=0, \text { in } S_{s} \\
& \|M\|_{\gamma}=0, \quad\|N\|_{\gamma}=0, \text { on } \Gamma_{\gamma}
\end{aligned}
$$

$$
\begin{gathered}
\left\|\sigma_{i j} n_{j}\right\|_{\gamma}=-\left\|C_{i j \alpha 3}\right\|_{\gamma} n_{j}, \\
\left\|D_{j} n_{j}\right\|_{\gamma}=-\left\|e_{j \alpha 3} \quad\right\|_{\gamma} n_{j}, \text { on } \Gamma_{\gamma} \\
\langle M\rangle=\langle N\rangle=0
\end{gathered}
$$

where $\quad \sigma_{i j}^{(s)}=C_{i j k k}^{(s)} M_{, k}^{(s)}+e_{k i j}^{(s)} N_{, k}^{(s)}, \quad D_{j}^{(s)}=e_{j 3 k}^{(s)} M_{, k}^{(s)}-$ $\kappa_{k j}^{(s)} N_{, k}^{(s)} ; M$ and $N$ are the local functions corresponding to the mechanical displacements and the electric potentials associated to the present local problems with $\alpha=1,2$.

Thus, the functions $M$ and $N$ are sought in such a way that they also are doubly periodic harmonic functions of the complex variable $z=y_{1}+i y_{2}$ in the rhombic periodic cell $S$ with the periods $w_{1}=1$ and $w_{2}=e^{i \theta}$, $0<\theta \leq \pi / 2$ (see Figure 2).

\section{Local problems solution ${ }_{\alpha 3} L(\alpha=\mathrm{I}, 2)$}

Considering the above mathematical statement, the functions $M^{(1)}, \mathrm{N}^{(1)}$ and $M^{(2)}, \mathrm{N}^{(2)}$ are to be found in terms of the Laurent expansions of doubly periodic harmonic functions over the region $S_{1}$ (matrix) and $S_{2}$ (fiber), respectively, as in (20). In the interphase $S_{I}$ we take the expansions

$$
\begin{gathered}
M^{(I)}(z)=\operatorname{Re}\left\{\sum_{p=1}^{\infty}{ }^{o} a_{-p}^{(I)} \frac{R_{1}^{p}}{z^{p}}+\sum_{p=1}^{\infty}{ }^{o} a_{p}^{(I)} \frac{z^{p}}{R^{p}}\right\}, \\
N^{(I)}(z)=\operatorname{Re}\left\{\sum_{p=1}^{\infty}{ }^{o} b_{-p}^{(I)} \frac{R_{1}^{p}}{z^{p}}+\sum_{p=1}^{\infty}{ }^{o} b_{p}^{(I)} \frac{z^{p}}{R^{p}}\right\}
\end{gathered}
$$

where the symbol $R e$ is the real part of the complex number. $R$ and $R_{1}\left(R_{1}=R+t\right)$ are the inner and outer radius of the interphase and $t$ is the thickness of the interphase in the composite (see, Figure 2). $a_{p}^{(I)}, a_{-p}^{(I)}, b_{p}^{(I)}, b_{-p}^{(I)}$ are complex undetermined coefficients that depend on the local problems ${ }_{\alpha 3} L$ to be solved and the superscript " $o$ " next to the summation symbol means that " $p$ " runs only over odd integers.

Substituting the expansions (20) and (33) into the contact conditions at the interface (30)-(31), after some algebraic manipulations, we obtain a system (see Appendix 1, (57), (58), (61) and (62)) of eight equations with the unknown constants $\bar{a}_{p}, a_{p}^{(I)}, \bar{a}_{-p}^{(I)}, \bar{b}_{p}, b_{p}^{(I)}, \quad \bar{b}_{-p}^{(I)}, \quad c_{p}$ and $d_{p}$. This system can be rewritten into a new matricial system in terms of the conjugate of $\bar{a}_{p}$ and $\bar{b}_{p}$, which can be solved by Gauss's method. Once we have the unknown constants, we can determine the effective coefficients as given in the following section.

The local problem ${ }_{\alpha} I(\alpha=1,2)$ can be solved analogously and the associated effective coefficients can be determined. 


\section{Effective coefficients}

The effective coefficients related to the local problems ${ }_{\alpha 3} L$ are given in this section.

Effective coefficients associate with the local problem ${ }_{13} L$

$$
\begin{aligned}
C_{1313}^{*} & =\left\langle C_{1313}\right\rangle+\left\langle C_{1331} M, 1\right\rangle+\left\langle e_{113} N_{, 1}\right\rangle, \\
C_{2313}^{*} & =\left\langle C_{2332} M_{, 2}\right\rangle+\left\langle e_{223} N_{, 2}\right\rangle, \\
e_{113}^{*} & =\left\langle e_{113}\right\rangle+\left\langle e_{131} M_{, 1}\right\rangle-\left\langle\kappa_{11} N_{, 1}\right\rangle \\
e_{213}^{*} & =\left\langle e_{113} M_{, 2}\right\rangle-\left\langle\kappa_{11} N_{, 2}\right\rangle
\end{aligned}
$$

Effective coefficients associate with the local problem ${ }_{23} L$

$$
\begin{aligned}
C_{1323}^{*} & =\left\langle C_{1331} M_{, 1}\right\rangle+\left\langle e_{113} N_{, 1}\right\rangle, \\
C_{2323}^{*} & =\left\langle C_{1313}\right\rangle+\left\langle C_{1313} M_{, 2}\right\rangle+\left\langle e_{113} N_{, 2}\right\rangle, \\
e_{123}^{*} & =\left\langle e_{131} M_{, 1}\right\rangle-\left\langle\kappa_{11} N_{, 1}\right\rangle, \\
e_{223}^{*} & =\left\langle e_{223}\right\rangle+\left\langle e_{113} M_{, 2}\right\rangle-\left\langle\kappa_{11} N_{, 2}\right\rangle
\end{aligned}
$$

Consequently, the local functions $M$ and $N$ are the local problems solutions ${ }_{\alpha 3} L$, respectively. The expressions of the effective coefficients (34)-(35) are transformed applying Green's theorem to the area integrals. Subsequently, substituting expansions (20) and (33) into the line integrals and using the orthogonality of the set functions $\{\cos (n x), \sin (n x)\}_{n=-\infty}^{\infty}$ in $[0,2 \pi]$, the dimensionless effective properties analytical expressions are only obtained as functions of the unknown constants $\bar{a}_{1}$ and $\bar{b}_{1}$ associated to each local problems ${ }_{\alpha 3} L,{ }_{\alpha} I(\alpha=1,2)$ as follows

a) local problem ${ }_{13} L$ b) local problem ${ }_{23} L$

$$
\begin{aligned}
C_{1323}^{*}- & i C_{2323}^{*}=-i\left(C_{1313}\right)_{v}+\left(\chi_{1}-1\right) \frac{\left(V_{I}+V_{2}\right)}{R_{1}} \\
& \times\left[a_{0}+\sum_{k=1}^{o}{ }^{o} a_{k} \eta_{k 1}+\bar{a}_{1}\right] \\
& +\left(\chi_{2}-\chi_{1}\right) \frac{V_{2}}{R} c_{1}+ \\
& +\left(E_{15}^{(I)} \sqrt{\chi_{1} \kappa_{1}}-E_{15}^{(1)}\right) \frac{\left(V_{I}+V_{2}\right)}{R_{1}} \\
& \times\left[b_{0}+\sum_{k=1}^{\infty} b_{k} \eta_{k 1}+\bar{b}_{1}\right] \\
& +\left(E_{15}^{(2)} \chi_{2}-E_{15}^{(2)} \sqrt{\frac{\chi_{1} \chi_{2} \kappa_{1}}{\kappa_{2}}}\right) \frac{V_{2}}{R} d_{1}, \\
e_{123}^{*}-i e_{223}^{*}= & -i\left(e_{113}\right)_{v}+\left(E_{15}^{(I)} \sqrt{\chi_{1} \kappa_{1}}-E_{15}^{(1)}\right) \frac{\left(V_{I}+V_{2}\right)}{R_{1}} \\
& \times\left[a_{0}+\sum_{k=1}^{\infty}{ }^{o} a_{k} \eta_{k 1}+\bar{a}_{1}\right] \\
& +\left(E_{15}^{(2)} \sqrt{\chi_{2} \kappa_{2}}-E_{15}^{(I)} \sqrt{\chi_{1} \kappa_{1}}\right) \frac{V_{2}}{R} c_{1}- \\
& -\left(\kappa_{1}-1\right) \frac{\left(V_{I}+V_{2}\right)}{R_{1}}\left[b_{0}+\sum_{k=1}^{\infty} b_{k} \eta_{k 1}+\bar{b}_{1}\right] \\
& -\left(\sqrt{\chi_{2} \kappa_{2}}-\sqrt{\frac{\chi_{2} \kappa_{1} \kappa_{1}}{\kappa_{2}}}\right) \frac{V_{2}}{R} d_{1}
\end{aligned}
$$

$$
\begin{aligned}
C_{1313}^{*}- & i C_{2313}^{*}=\left(C_{1313}\right)_{v}+\frac{\left(V_{I}+V_{2}\right)}{R_{1}}\left(\chi_{1}-1\right)\left[a_{0}+\sum_{k=1}^{\infty}{ }^{o} a_{k} \eta_{k 1}\right]+\frac{\left(V_{I}+V_{2}\right)}{R_{1}}\left(\chi_{1}-1\right) \bar{a}_{1}+\frac{V_{2}}{R}\left(\chi_{2}-\chi_{1}\right) c_{1}+ \\
& +\frac{\left(V_{I}+V_{2}\right)}{R_{1}}\left(E_{15}^{(I)} \sqrt{\chi_{1} \kappa_{1}}-E_{15}^{(1)}\right)\left[b_{0}+\sum_{k=1}^{o} b_{k} \eta_{k 1}\right]+\frac{\left(V_{I}+V_{2}\right)}{R_{1}}\left(E_{15}^{(I)} \sqrt{\chi_{1} \kappa_{1}}-E_{15}^{(1)}\right) \bar{b}_{1} \\
& +\frac{V_{2}}{R}\left(E_{15}^{(2)} \chi_{2}-E_{15}^{(I)} \sqrt{\frac{\chi_{1} \chi_{2} \kappa_{1}}{\kappa_{2}}}\right) d_{1}, \\
e_{113}^{*}-i e_{213}^{*}= & \left(e_{113}\right)_{v}+\frac{\left(V_{I}+V_{2}\right)}{R_{1}}\left(E_{15}^{(I)} \sqrt{\chi_{1} \kappa_{1}}-E_{15}^{(1)}\right)\left[a_{0}+\sum_{k=1}^{\infty}{ }^{*} a_{k} \eta_{k 1}\right]+\frac{\left(V_{I}+V_{2}\right)}{R_{1}}\left(E_{15}^{(I)} \sqrt{\chi_{1} \kappa_{1}}-E_{15}^{(1)}\right) \bar{a}_{1}+ \\
& +\frac{V_{3}}{R}\left(E_{15}^{(2)} \sqrt{\chi_{2} \kappa_{2}}-E_{15}^{(I)} \sqrt{\chi_{1} \kappa_{1}}\right) c_{1}-\frac{\left(V_{I}+V_{2}\right)}{R_{1}}\left(\kappa_{1}-1\right)\left[b_{0}+\sum_{k=1}^{\infty}{ }^{*} b_{k} \eta_{k 1}\right] \\
& -\frac{\left(V_{I}+V_{2}\right)}{R_{1}}\left(\kappa_{1}-1\right) \overline{b_{1}}-\frac{V_{3}}{R}\left(\sqrt{\chi_{2} \kappa_{2}}-\sqrt{\frac{\chi_{2} \kappa_{1} \kappa_{1}}{\kappa_{2}}}\right) d_{1}
\end{aligned}
$$


c) local problem ${ }_{1} I$

$$
\begin{aligned}
& e_{113}^{*}-i e_{123}^{*}=\left(e_{113}\right)_{v}+\frac{\left(V_{I}+V_{2}\right)}{R_{1}} \sqrt{C_{1313}^{(1)} \kappa_{11}^{(1)}} \\
& \times\left[\left(\chi_{1}-1\right) \bar{a}_{1}+\left(E_{15}^{(I)} \sqrt{\chi_{1} \kappa_{1}}-E_{15}^{(1)}\right) \bar{b}_{1}\right]+ \\
& +\frac{\left(V_{I}+V_{2}\right)}{R_{1}}\left(\chi_{1}-1\right) \sqrt{C_{1313}^{(1)} \kappa_{11}^{(1)}} \\
& \times\left[a_{0}+\sum_{k=1}^{\infty}{ }^{o} a_{k} \eta_{k 1}\right]+\frac{V_{2}}{R} \sqrt{C_{1313}^{(1)} \kappa_{11}^{(1)}} \\
& \times\left(\sqrt{\chi_{2} \kappa_{2}}-\sqrt{\frac{\chi_{1} \chi_{1} \kappa_{2}}{\chi^{(2)} *}}\right) c_{1}+\frac{\left(V_{I}+V_{2}\right)}{R_{1}} \\
& \times\left(E_{15}^{(I)} \sqrt{\chi_{1} \kappa_{1}}-E_{15}^{(1)}\right) \sqrt{C_{1313}^{(1)} \kappa_{11}^{(1)}} \\
& \times\left[b_{0}+\sum_{k=1}^{\infty}{ }^{o} b_{k} \eta_{k 1}\right]+\frac{V_{2}}{R} \sqrt{C_{1313}^{(1)} \kappa_{11}^{(1)}} \\
& \times\left(E_{15}^{(2)} \sqrt{\chi_{2} \kappa_{2}}-E_{15}^{(I)} \sqrt{\chi_{1} \kappa_{1}}\right) d_{1}, \\
& \kappa_{11}^{*}-i \kappa_{21}^{*}=\left(\kappa_{11}\right)_{v}-\frac{\left(V_{I}+V_{2}\right)}{R_{1}} \kappa_{11}^{(1)}\left[\left(E_{15}^{(I)} \sqrt{\chi_{1} \kappa_{1}}-E_{15}^{(1)}\right)\right. \\
& \left.\times \bar{a}_{1}-\left(\kappa_{1}-1\right) \bar{b}_{1}\right]-\frac{\left(V_{I}+V_{2}\right)}{R_{1}} \kappa_{11}^{(1)} \\
& \times\left(E_{15}^{(I)} \sqrt{\chi_{1} \kappa_{1}}-E_{15}^{(1)}\right)\left[a_{0}+\sum_{k=1}^{\infty}{ }^{o} a_{k} \eta_{k 1}\right] \\
& -\kappa_{11}^{(1)} \frac{V_{2}}{R}\left(E_{15}^{(2)} \kappa_{2}-E_{15}^{(I)} \sqrt{\frac{\chi_{1} \kappa_{1} \kappa_{2}}{\chi_{2}}}\right) c_{1}+ \\
& +\frac{\left(V_{I}+V_{2}\right)}{R_{1}} \kappa_{11}^{(1)}\left(\kappa_{1}-1\right)\left[b_{0}+\sum_{k=1}^{\infty}{ }^{o} b_{k} \eta_{k 1}\right] \\
& +\frac{V_{2}}{R} \kappa_{11}^{(1)}\left(\kappa_{2}-\kappa_{1}\right) d_{1}
\end{aligned}
$$

d) local problem ${ }_{2} I$

$$
\begin{aligned}
e_{213}^{*}- & i e_{223}^{*}=-i\left(e_{223}\right)_{v}+\frac{\left(V_{I}+V_{2}\right)}{R_{1}}\left(\chi_{1}-1\right) \\
& \times \sqrt{C_{1313}^{(1)} \kappa_{11}^{(1)}}\left[a_{0}+\sum_{k=1}^{\infty}{ }^{o} a_{k} \eta_{k 1}+\bar{a}_{1}\right] \\
& +\frac{V_{2}}{R} \sqrt{C_{1313}^{(1)} \kappa_{11}^{(1)}}\left(\sqrt{\chi_{2} \kappa_{2}}-\sqrt{\frac{\chi_{1} \chi_{1} \kappa_{2}}{\chi_{2}}}\right) c_{1}+ \\
& +\frac{\left(V_{I}+V_{2}\right)}{R_{1}}\left(E_{15}^{(2)} \sqrt{\chi_{1} \kappa_{1}}-E_{15}^{(1)}\right) \sqrt{C_{1313}^{(1)} \kappa_{11}^{(1)}} \\
& \times\left[b_{0}+\sum_{k=1}^{\infty}{ }^{o} b_{k} \eta_{k 1}+\bar{b}_{1}\right]+\frac{V_{2}}{R} \sqrt{C_{1313}^{(1)} \kappa_{11}^{(1)}} \\
& \times\left(E_{15}^{(3)} \sqrt{\chi_{2} \kappa_{2}}-E_{15}^{(2)} \sqrt{\chi_{1} \kappa_{1}}\right) d_{1},
\end{aligned}
$$

$$
\begin{aligned}
\kappa_{12}^{*}-i \kappa_{22}^{*}= & -i\left(\kappa_{22}\right)_{v}-\frac{\left(V_{I}+V_{2}\right)}{R_{1}} \kappa_{11}^{(1)}\left(E_{15}^{(I)} \sqrt{\chi_{1} \kappa_{1}}-E_{15}^{(1)}\right) \\
& \times\left[a_{0}+\sum_{k=1}^{\infty}{ }^{o} a_{k} \eta_{k 1}+\bar{a}_{1}\right]-\frac{V_{2}}{R} \kappa_{11}^{(1)} \\
& \times\left(E_{15}^{(2)} \kappa_{2}-E_{15}^{(I)} \sqrt{\frac{\chi_{1} \kappa_{1} \kappa_{2}}{\chi_{2}}}\right) c_{1}+\frac{\left(V_{I}+V_{2}\right)}{R_{1}} \\
& \times\left(\kappa_{1}-1\right) \kappa_{11}^{(1)}\left[b_{0}+\sum_{k=1}^{\infty}{ }^{o} b_{k} \eta_{k 1}+\bar{b}_{1}\right] \\
& +\frac{V_{2}}{R} \kappa_{11}^{(1)}\left(\kappa_{2}-\kappa_{1}\right) d_{1}
\end{aligned}
$$

The notation $(f)_{v}=f^{(1)} V_{1}+f^{(I)} V_{I}+f^{(2)} V_{2}$, where $V_{1}, V_{I}$ and $V_{2}$ are the volume fraction per unit length occupied by the matrix, interphase and the fiber, respectively is used. Moreover, $V_{1}+V_{I}+V_{2}=1$ and $V=\left\|w_{1}\right\|\left\|w_{2}\right\| \sin \theta$ represent the periodic cell volume. The expressions (36)-(39) of the effective coefficients depend on the material properties, volume fractions and the unknown constants $a_{k}, b_{k}, c_{1}$ and $d_{1}$. The unknown constants can be found from the system obtained in Appendix 1.

\section{Finite element approach}

Different approaches can be used for the determination of the effective coefficients of composites. This section describes the approach of using the representative volume element concept in combination with the FEM in order to evaluate the effective behavior of piezoelectric composites. Since the considered composite is periodic, the representative volume element can be described by a periodic repeatable unit cell. Under the assumption of linear piezoelectricity, we consider a composite consists of two phases, where

$$
\begin{array}{r}
\frac{\partial \sigma_{i j}^{\gamma}}{\partial \tilde{y}_{j}}=0, \quad \frac{\partial D_{i}^{\gamma}}{\partial \tilde{y}_{i}}=0, \quad \tilde{\mathbf{y}} \in S, \quad \sigma_{i j}^{\gamma}=C_{i j k l}^{\gamma} \varepsilon_{k l}^{\gamma}-e_{k i j}^{\gamma} E_{k}^{\gamma}, \\
D_{i}^{\gamma}=e_{i k l}^{\gamma} \varepsilon_{k l}^{\gamma}+\kappa_{i k}^{\gamma} E_{k}^{\gamma}, \quad i, j, k, l=1,2,3, \quad \gamma=m, f
\end{array}
$$

holds in each phase. The quantities $\sigma_{i j}^{\gamma}, \varepsilon_{k l}^{\gamma}, D_{i}^{\gamma}, E_{j}^{\gamma}, C_{i j k l}^{\gamma}$ and $e_{i k l}^{\gamma}$ and $\kappa_{i j}^{\gamma}$ are the stress components, the strain components, the electric flux density components, the electric field intensity components, the elasticity coefficients, the piezoelectric coefficients and the dielectric coefficients related to phase $\gamma$, respectively. Moreover, the strain tensor and the electric field intensity must satisfy the following relation

$$
\varepsilon_{i j}^{\gamma}=\frac{1}{2}\left(\frac{\partial u_{i}^{\gamma}}{\partial \tilde{y}_{j}}+\frac{\partial u_{j}^{\gamma}}{\partial \tilde{y}_{i}}\right), \quad E_{i}^{\gamma}=-\frac{\partial \phi^{\gamma}}{\partial \tilde{y}_{i}}
$$


where $u_{i}^{\gamma}$ and $\phi^{\gamma}$ are the displacement components and the electric potential, respectively. The connectivity of the phases is given by the imperfect conditions

$$
\begin{array}{ll}
\sigma_{r r}^{m}=\sigma_{r r}^{f}=\tilde{K}_{r}\left(u_{r}^{m}-u_{r}^{f}\right), & \sigma_{r \vartheta}^{m}=\sigma_{r \vartheta}^{f}=\tilde{K}_{\vartheta}\left(u_{\vartheta}^{m}-u_{\vartheta}^{f}\right), \\
\sigma_{r z}^{m}=\sigma_{r z}^{f}=\tilde{K}_{z}\left(u_{z}^{m}-u_{z}^{f}\right), & D_{r}^{m}=D_{r}^{f}=\tilde{K}_{\phi}\left(\phi^{f}-\phi^{m}\right)
\end{array}
$$

which are related to a cylindrical coordinate system $\{r, \vartheta, z\}$. Here the imperfect parameters $\tilde{K}_{i}, \mathrm{i}=\mathrm{r}, \vartheta, \mathrm{z}$ and $\tilde{K}_{\phi}$ characterize the stiffness of the interface expressed by force per volume for elastic part and capacitance per area for electric part. In general, these parameters are unknown. These interfacial conditions imply continuity in the stresses and in the electric flux density. On the other hand, the displacements and the electric potential are discontinuous.

Under specific assumptions the imperfect parameters can be derived from a three-phase composite model. The material properties and the thickness of the third phase, which is the interphase between the fiber and the matrix, characterize the imperfect contact parameters. In the case of a pure elastic problem and under the assumptions of a very thin thickness and flexible isotropic material properties of the interphase Hashin ${ }^{29}$ derived the following relations

$$
\begin{aligned}
\tilde{K}_{r} & =\frac{E^{I}\left(1-v^{I}\right)}{\left(1+v^{I}\right)\left(1-2 v^{I}\right) t}, \quad \tilde{K}_{\vartheta}=\frac{E^{I}}{2\left(1+v^{I}\right) t}, \\
\tilde{K}_{z} & =\frac{E^{I}}{2\left(1+v^{I}\right) t}
\end{aligned}
$$

where $E^{I}$ and $v^{I}$ are the Young's modulus and the Poisson's ratio of the interphase, respectively. For the assumed isotropy of the interphase the expressions in (43) are identical with $\tilde{K}_{r}=C_{11}^{I} / t, \tilde{K}_{\vartheta}=C_{44}^{I} / t=\tilde{K}_{z}$. Analogously, considering piezoelectric three-phase composites the electrical imperfect contact parameter $\tilde{K}_{\phi}$ is given by

$$
\tilde{K}_{\phi}=\kappa_{11}^{I} / t
$$

where $\kappa_{11}^{I}$ is a dielectric coefficient of the dielectric second-order tensor of the interphase, which is assumed to have material properties with elastic and dielectric isotropy and no piezoelectric capabilities. The thickness of the interphase can be derived from the ratio $\eta=t / R$ where $R$ is the radius of the fibers. Later in the calculations and in the analysis of results, this ratio will have a fixed value.

In order to derive effective properties of a two-phase composite with imperfect interface stated by the equilibrium problem (40) and the imperfect contact

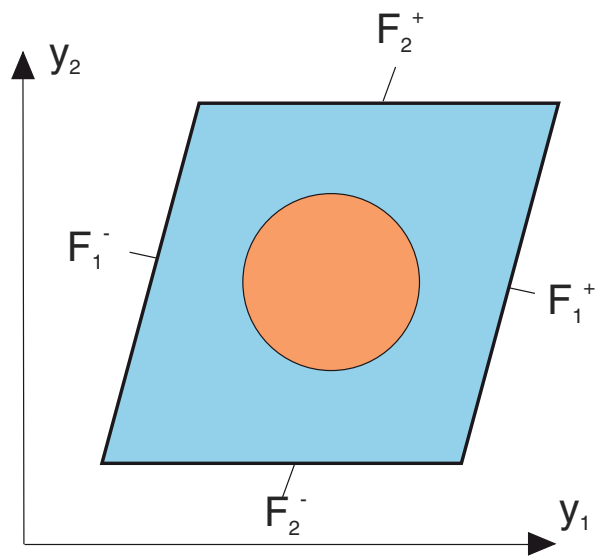

Figure 3. Notation of the boundaries related to the cross section of the finite element implementation.

conditions (42), appropriate boundary conditions on the periodic unit cell have to be applied. It is assumed that the displacements and the electric potential on the cell boundary are a superposition of a linear and a periodic part

$$
u_{i}=\varepsilon_{i j}^{0} \tilde{y}_{j}+u_{i}^{p e r}, \quad \phi=-E_{i}^{0} \tilde{y}_{i}+\phi^{p e r}
$$

where $\varepsilon_{i j}^{0}$ and $E_{i}^{0}$ are given quantities. Considering the differences on opposite boundary faces of $u_{i}$ and $\phi$ constraint equations of the form

$u_{i}^{F_{k}^{+}}-u_{i}^{F_{k}^{-}}=\varepsilon_{i j}^{0}\left(\tilde{y}_{j}^{F_{k}^{+}}-\tilde{y}_{j}^{F_{k}^{-}}\right), \quad \phi^{F_{k}^{-}}-\phi^{F_{k}^{+}}=E_{i}^{0}\left(\tilde{y}_{i}^{F_{k}^{+}}-\tilde{y}_{i}^{F_{k}^{-}}\right)$

can be derived (see Figure 3).

Using the analogous assumption as for the elastic case related to perfect bonding of phases given previously, the averages of the strains, stresses, electric fluxes and the electric fields representing composite quantities at macroscopic level are given by

$$
\begin{aligned}
\left\langle\sigma_{i j}\right\rangle & =\frac{1}{|S|} \int_{\partial S} \sigma_{i k} n_{k} \tilde{y}_{j} \mathrm{~d} A, & \left\langle\varepsilon_{i j}\right\rangle & =\frac{1}{|S|} \int_{\partial S}\left(u_{i} n_{j}+u_{j} n_{i}\right) \mathrm{d} A, \\
\left\langle D_{i}\right\rangle & =\frac{1}{|S|} \int_{\partial S} D_{k} n_{k} \tilde{y}_{i} \mathrm{~d} A, & \left\langle E_{i}\right\rangle & =-\frac{1}{|S|} \int_{\partial S} \phi n_{i} \mathrm{~d} A
\end{aligned}
$$

The effective coefficients of the composite can be derived from the relations

$$
\left\langle\sigma_{i j}\right\rangle=C_{i j k l}^{*}\left\langle\varepsilon_{k l}\right\rangle-e_{k i j}^{*}\left\langle E_{k}\right\rangle, \quad\left\langle D_{i}\right\rangle=e_{i k l}^{*}\left\langle\varepsilon_{k l}\right\rangle+\kappa_{i j}^{*}\left|E_{j}\right\rangle
$$

Equation (48) represents the constitutive law of the homogenized composite material. 
Using the equation (45) and the knowledge of the continuity or discontinuity of quantities across the phase interface, the following relations hold

$$
\begin{aligned}
\left\langle\sigma_{i j}\right\rangle= & \frac{1}{|S|} \int_{S^{m}} \sigma_{i j} \mathrm{~d} V+\frac{1}{|S|} \int_{S^{f}} \sigma_{i j} \mathrm{~d} V, \\
\left\langle\varepsilon_{i j}\right\rangle= & \frac{1}{|S|} \int_{S^{m}} \varepsilon_{i j} \mathrm{~d} V+\frac{1}{|S|} \int_{S^{m}} \varepsilon_{i j} \mathrm{~d} V \\
& +\frac{1}{|S|} \int_{\Gamma}\left(\left\|u_{i}\right\| n_{j}+\left\|u_{j}\right\| n_{i}\right) \mathrm{d} A=\varepsilon_{i j}^{0}, \\
\left\langle D_{i}\right\rangle= & \frac{1}{|S|} \int_{S^{m}} D_{i} \mathrm{~d} V+\frac{1}{|S|} \int_{S^{f}} D_{i} \mathrm{~d} V, \\
\left\langle E_{i}\right\rangle= & \frac{1}{|S|} \int_{S^{m}} E_{i} \mathrm{~d} V+\frac{1}{|S|} \int_{S^{f}} E_{i} \mathrm{~d} V \\
& -\frac{1}{|S|} \int_{\Gamma}\|\phi\| n_{i} \mathrm{~d} A=E_{i}^{0}
\end{aligned}
$$

where $\|\cdot\|$ denotes the difference of a quantity . between the matrix and the fiber phase on the interface $\Gamma$, i.e. $\left\|u_{i}\right\|=u_{i}^{m}-u_{i}^{f}$. The volume of the RVE is denoted as $|S|$. By setting the components $\varepsilon_{i j}^{0}$ and $E_{i}^{0}$ of the tensors $\boldsymbol{\varepsilon}^{0}$ (second order) and $\mathbf{E}^{0}$ (first order) in equation (45) to appropriate values, the effective properties of the composite can easily derived from equation (48) by separating the dependency of $\left\langle\sigma_{i j}\right\rangle,\left\langle\varepsilon_{i j}\right\rangle,\left\langle D_{i}\right\rangle$ and $\left\langle E_{i}\right\rangle$. This setting is divided into nine cases. In each case, nine effective coefficients can be determined. These cases are

$$
\begin{aligned}
\boldsymbol{\varepsilon} c^{0} \in & \left\{\begin{array}{lll}
a & 0 & 0 \\
0 & 0 & 0 \\
0 & 0 & 0
\end{array}\right),\left(\begin{array}{lll}
0 & 0 & 0 \\
0 & a & 0 \\
0 & 0 & 0
\end{array}\right),\left(\begin{array}{ccc}
0 & 0 & 0 \\
0 & 0 & 0 \\
0 & 0 & a
\end{array}\right), \\
& \left(\begin{array}{ccc}
0 & 0 & 0 \\
0 & 0 & 0.5 a \\
0 & 0.5 a & 0
\end{array}\right),\left(\begin{array}{ccc}
0 & 0 & 0.5 a \\
0 & 0 & 0 \\
0.5 a & 0 & 0
\end{array}\right), \\
& \left.\left(\begin{array}{ccc}
0 & 0.5 a & 0 \\
0.5 a & 0 & 0 \\
0 & 0 & 0
\end{array}\right)\right\}
\end{aligned}
$$

while

$$
\mathbf{E}^{0}=(0,0,0)^{T}
$$

and the three cases

$$
\mathbf{E}^{0} \in\left\{\left(\begin{array}{l}
d \\
0 \\
0
\end{array}\right),\left(\begin{array}{l}
0 \\
d \\
0
\end{array}\right),\left(\begin{array}{l}
0 \\
0 \\
d
\end{array}\right)\right\}
$$

while

$$
\boldsymbol{\varepsilon}^{0}=\left(\begin{array}{lll}
0 & 0 & 0 \\
0 & 0 & 0 \\
0 & 0 & 0
\end{array}\right)
$$

Here the parameters $a$ and $d$ are arbitrarily chosen constants, where the first is dimensionless and the second has the same unit as the electric field.

The commercial software ANSYS is used for the numerical calculations using the FEM. The FE-model of the RVE is discretized by linear three-dimensional SOLID5 elements. Eight nodes and four degrees of freedom at each node characterize these elements. The discretized finite element model has a rhombic-shaped cross section. By choosing the edge lengths of the rhombic and the included angle $\theta$, the fiber distribution of the composite can be changed. To apply constraint equations, given in equation (46), for nodal pairs between opposite faces of the RVE, identical discretization is necessary. In addition, the surface regions of the phases, which intersection is denoted as $\Gamma$, have also a coincident finite element discretization. Taking into account the coincident nodal pairs on the intersection, spring elements and capacitor elements are used to simulate the imperfect contact conditions (see Figure 4). Per nodal pair, three one-dimensional COMBIN14 elements (in $r, \vartheta$ and $z$ directions) and one CIRCU94 element are defined. By this numerical implementation, the imperfect contact conditions can be represented by

$$
\begin{aligned}
& F_{r}=\hat{K}_{r}\left\|u_{r}\right\|, \quad F_{\vartheta}=\hat{K}_{\vartheta}\left\|u_{\vartheta}\right\|, \quad F_{z}=\hat{K}_{z}\left\|u_{z}\right\|, \\
& Q=\hat{K}_{\phi}\|\phi\|
\end{aligned}
$$

where the force components $F_{i}, i=r, \vartheta, z$ and the charge $Q$ are proportional to the differences of the displacement components and the difference of the electric potential, respectively. The parameters $\hat{K}_{i}$ represent the typical spring constants with force per length, $\hat{K}_{\phi}$ represents the capacitance. In order to get $\hat{K}_{i}$ from the parameters $\tilde{K}_{i}$ in equation (42) they are multiplied by a certain attached area $A_{n}$ related to the node $n$, where the spring is mounted like illustrated in Figure 5

$$
\hat{K}_{i}=\tilde{K}_{i} A_{n}
$$

Because of the discretization with linear elements, the cylindrical fiber becomes a prism with $n_{c s}$ faces, where $n_{c S}$ is the number of nodes in the circumferential direction. For an equidistant finite element discretization 

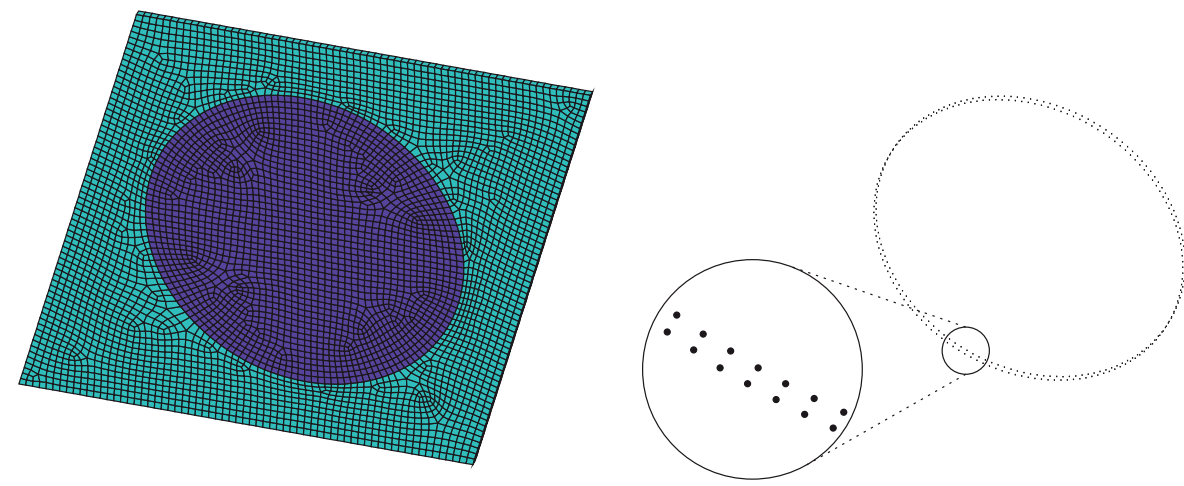

Figure 4. A discretized RVE with a rhombic-shaped cross section (left) and highlighted spring and capacitor elements (right) on the intersection of the phases.

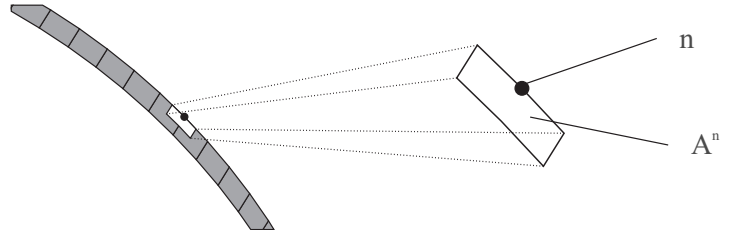

Figure 5. Attached area $A_{n}$ to node $n$ for calculation of spring constants.

and one element in longitudinal direction, $A_{n}$ can be calculated by

$$
A_{n}=R q \sin \left(\frac{\pi}{n_{c s}}\right)
$$

Here $R$ and $q$ are the radius of the fiber and the depth of the prism (longitudinal direction), respectively. Due to the finite element discretization, the integral formulations of the macroscopic quantities in equation (48) are changed to numerical ones. This means that the volume integrals become sums over elements, where the element volume multiplies each summand. The surface integrals are replaced by a summation of nodal displacement differences multiplied by factors depending on the surface geometry.

\section{Analysis of results}

In many real fiber-reinforced composites, the fibermatrix adhesion is imperfect. Linear spring and capacitor interface model (present approach) and interphase models are often used to simulate the adhesion behavior of reinforced composite materials. The aim of this section is to point out the effects of the fiber-matrix bond on the piezoelectric properties of composite materials based on the AHM and FEM. To date, to the author's knowledge, the problems associated to the calculations of effective properties for piezoelectric composite materials with defective interface conditions are very scarce in the literature. Some numerical results derived from the present model are given in order to illustrate the effect of the failure at the interface. In the context of the present study, variations of the spring stiffness and electric capacitor from full debonding and complete electric barrier to a perfect bonding interface are considered. There are certain scenarios associated with the full debonding interfaces for which the cylindrical inhomogeneity becomes equivalent to a hole.

In the following, some numerical results and validations are presented for piezoelectric composites. The material parameters used in the numerical estimation are displayed in Table 1.

1. Two-phase composites with square and hexagonal cells are studied. Mechanical and electrical perfect contact conditions are considered as a validation of the present models. In Table 2 a comparison of the effective moduli $C_{44}^{*}, e_{15}^{*}, \kappa_{11}^{*}$ for a two-phase composite (PZT-7A/Epoxy 1) with perfect contact computed by AHM, eigenfunction expansion variational method (EEVM) $)^{30}$ taken from Table 2 in Yan et al. ${ }^{30}$ and the finite element values implemented in the present work for the spring-capacitor model but adequate to a particular case of perfect contact is shown. This finite element approach reproduces the same values reported in the publication. ${ }^{30}$ Moreover, the results are compared also with other implementation of FEM. ${ }^{32}$ The volume fractions of fiber and interphase are $V_{2}=0.6$ and $V_{I}=0.0012$, respectively. The dimensionless imperfect parameters $K_{n}=K_{t}=K_{s}=K_{\varphi}=10^{12}$ are used in $\mathrm{AHM}$ spring-capacitor and three-phase models. The properties of the interphase are calculated by (43) and (44) multiplied by $R$. This comparison validates the spring-capacitor and three-phase models.

2. A comparison between spring-capacitor and three phase models by AHM and FEM (spring-capacitor 
Table 2. Comparison of the effective properties using spring-capacitor and three-phase models via AHM with EEVM and FEM for two-phase composite (PZT-7A/Epoxy I) with perfect adhesion.

\begin{tabular}{|c|c|c|c|c|c|c|}
\hline & \multicolumn{3}{|c|}{ Square cell } & \multicolumn{3}{|c|}{ Hexagonal cell } \\
\hline & $\mathrm{C}_{44}^{*}(\mathrm{GPa})$ & $\mathrm{e}_{15}^{*}\left(C / \mathrm{m}^{2}\right)$ & $\kappa_{11}^{*}(n C / V m)$ & $\mathrm{C}_{44}^{*}(\mathrm{GPa})$ & $\mathrm{e}_{15}^{*}\left(\mathrm{C} / \mathrm{m}^{2}\right)$ & $\kappa_{11}^{*}(n C /$ tabl; $V m)$ \\
\hline AHM spring-capacitor & 6.673 & 0.05195 & 0.1575 & 6.324 & 0.04232 & 0.1467 \\
\hline AHM three-phase & 6.673 & 0.05195 & 0.1575 & 6.3245 & 0.04232 & 0.1467 \\
\hline EEVM & 6.673 & 0.051960 & 0.1576 & 6.324 & 0.04232 & 0.1467 \\
\hline $\mathrm{FEM}^{32}$ & 6.683 & 0.05204 & 0.1577 & 6.328 & 0.04244 & 0.1469 \\
\hline FEM & 6.643 & 0.05154 & 0.1571 & 6.298 & 0.04221 & 0.1469 \\
\hline
\end{tabular}

AHM: asymptotic homogenization method; FEM: finite element method.

model) is presented in Table 3 for a composite PZT5/Epoxy 2 with rhombic cells and interphase of Epoxy 1. In the numerical examples presented in the table, the fiber volume fraction is $V_{2}=0.5$, the angle of the rhombic cell is $\theta=45^{\circ}, 60^{\circ}, 75^{\circ}$ and two different parameters of the interphase $\eta=t / R=0.00001$ and $\eta=0.01$ are considered. In such composite the interphase mechanical and electrical properties have influence on the overall behavior. The properties of a thin layer represented by springs and capacitors allow the analysis of the influence of this interphase on the overall coefficients. In order to compare the spring-capacitor, three-phase models via AHM and FEM as well as the imperfect parameters are obtained as a function of the interphase properties as it is reported in (43) and (44) multiplied by $R$. Notice that the three-phase and spring-capacitor models coincide for all the effective properties as $\eta \rightarrow 0$.The two theoretical approaches, FEM for the spring-capacitor model and AHM (spring-capacitor and three-phase models), are consistent with the numerical values. It is well-known the global axial behavior in fibrous piezoelectric composites with periodic hexagonal cell under perfect contact condition is transversely isotropic; however, due to the oblique arrangment of the cell, the global behavior of this composite changes and it has a monoclinic symmetry so the effective coefficient $C_{45}^{*}, e_{14}^{*}$ and $\kappa_{12}^{*}$ are different from zero for the antiplane problem (see the results for angle of the periodic cell $=45^{\circ}$ and $75^{\circ}$ ).

3. Figure 6 shows a validation of the spring-capacitor model with perfect electrical contact $\left(K_{\phi}=\infty\right)$ between the fiber and matrix (PZT-5A/Epoxy 3) and three-phase model, both computed by AHM with square cell, and the recent model published by Tita et al. ${ }^{21}$ The interphase parameters are calculated by (43) multiplied by the fiber radius $R$ and the thickness is $\eta=10^{-3}$.In the report, ${ }^{21}$ a numerical approach is proposed to evaluate the effective properties of piezoelectric fibers embedded in a non-piezoelectric matrix, considering imperfect contact between the fiber and the matrix. A representative volume element is analyzed via FEM for different loadings with suitable boundary conditions. AHM approaches present a good concordance for the transversal effective coefficients $C_{44}^{*}, e_{15}^{*}, \kappa_{11}^{*}$ for low volume fraction up to 0.5 . A separation with the numerical computation using FEM (curves with "+") is notable for higher volume fraction. It would be explained as the accumulation of error due to the approximation for high volume fraction of the FEM. Moreover, a very good match is seen as $K_{t}=K_{s}=1$ for the effective properties $C_{44}^{*}$ and $e_{15}^{*}$. Total accuracy of the models is observed for the dielectric permittivity $\kappa_{11}^{*}$. All the curves overlap for different values of the parameter $K_{t}$, thus, the mechanical imperfect parameters do not influence the dielectric effective property $\kappa_{11}^{*}$. The composite becomes more flexible for the low values of the imperfection $K_{t}=K_{s}$ parameters and the high volume fraction of the fiber.

4. Figure 7 reveals the effect of the electrical imperfection $\left(K_{\phi} \ll \infty\right)$ in a two-phase composite (PZT-5A/ Epoxy 3) using the spring-capacitor model and a three-phase model via AHM with square cell for the transversal piezoelectric and dielectric permittivity effective coefficients $e_{15}^{*}$ and $\kappa_{11}^{*}$, respectively. The same values of mechanical imperfection $K_{t}$ used in the previous figures are considered. The interphase parameters are calculated by (43) and (44) multiplied by the fiber radius $R$ and the thickness of the interphase is $\eta=10^{-3}$. The global properties of $e_{15}^{*}$ and $\kappa_{11}^{*}$ display remarkable diminishing values when the imperfect electric parameter $K_{\phi}$ decreases. It ensures the influence of the electrical imperfect adhesion in the characterization of the piezoelectric composites. However, the numerical experiments realized in this work show that the electric imperfection does not affect the elastic constant in the composite.

5. Figure 8 shows the effective properties $C_{44}^{*}, e_{15}^{*}$ and $\kappa_{11}^{*}$ against $\log _{10}\left(G^{I} / G^{m}\right)$ or $\log _{10}\left(\kappa_{11}^{I} / \kappa_{11}^{m}\right)$, 


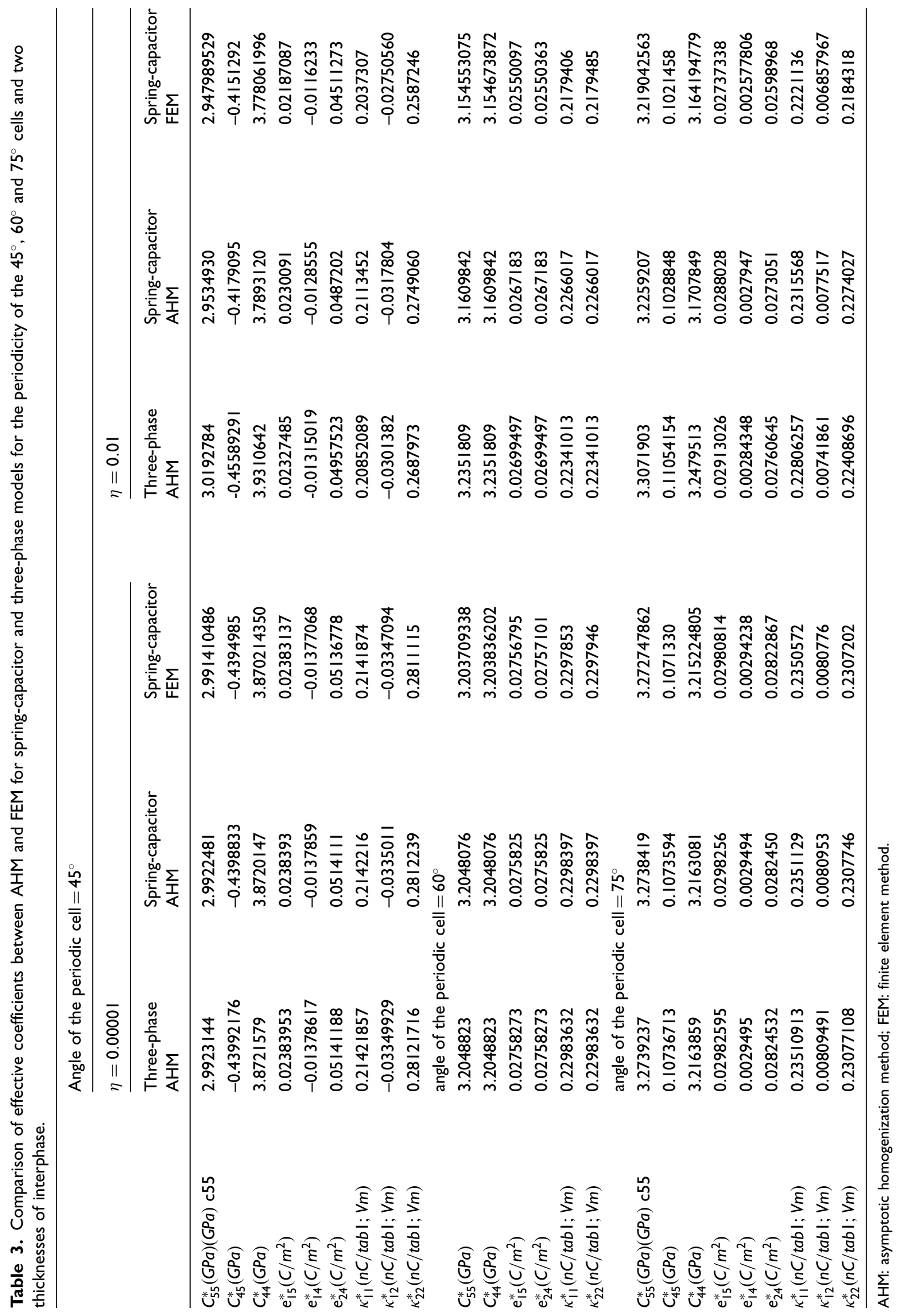



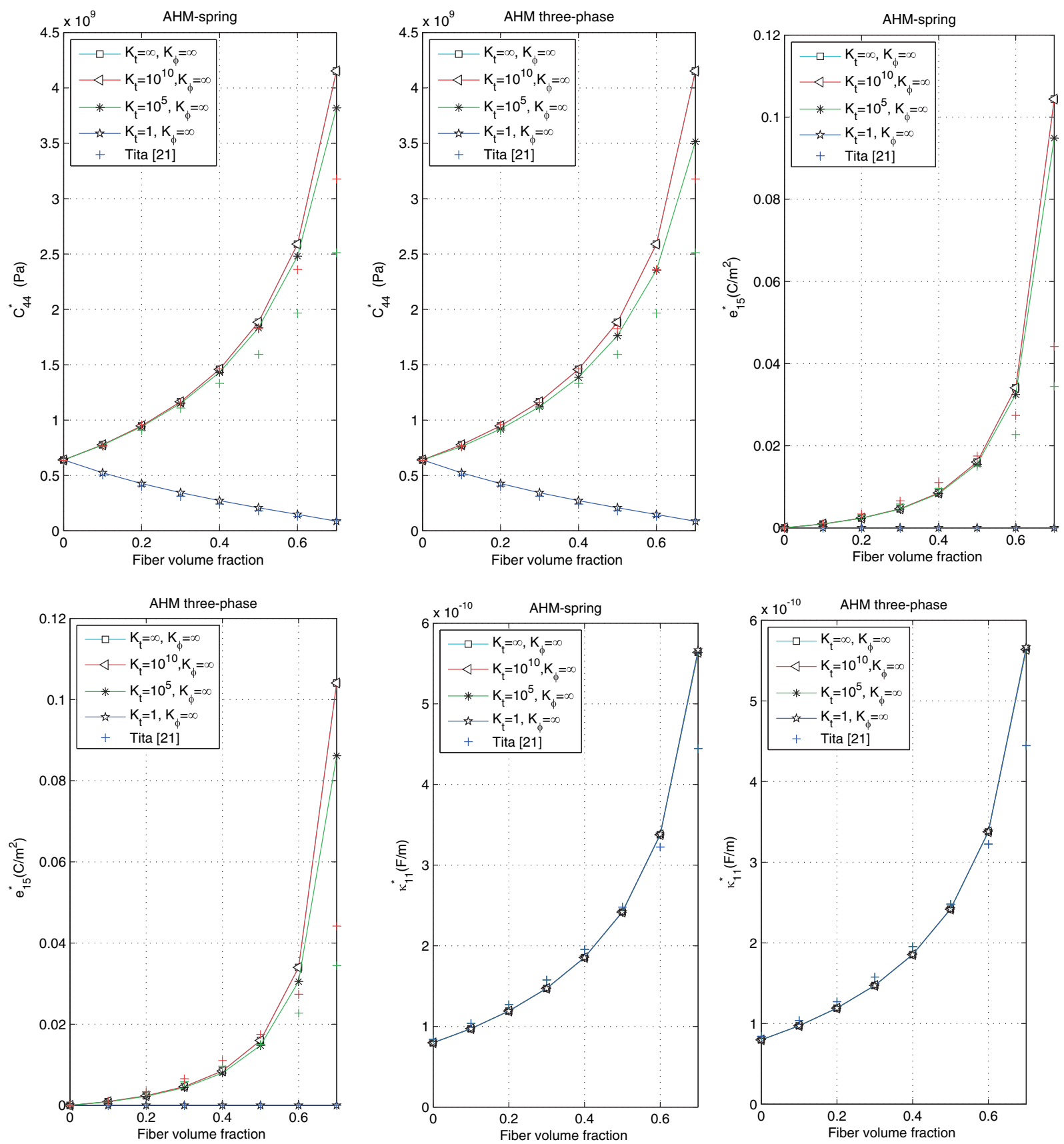

Figure 6. Behavior of the transverse effective coefficients $C_{44}^{*}, \mathrm{e}_{15}^{*}, \kappa_{1 \mid}^{*}$ derived by the spring-capacitor model (shown on the lefthand side of the figure) with perfect electric contact $\left(K_{\phi}=\infty\right)$ and the three-phase model (on the right-hand side of the figure) via AHM with square cell (solid line) and the numerical approach reported in Tita et al. ${ }^{21}$ (denoted by + ). Solid line and "+" with the same color correspond to the same imperfect parameters.

respectively, related to unidirectional periodic transversely isotropic composite with hexagonal cell. The parameters $G^{I}\left(G^{m}\right)$ and $\kappa_{11}^{I}\left(\kappa_{11}^{m}\right)$ are the shear and dielectric permittivity of the composite interphase (matrix). The composite is made of Epoxy 2 matrix with piezoelectric PZT-5 reinforcements. The physical characteristics are given in Table 1. In the computation the Poisson's ratio of interphase is fixed and equal to $v^{(I)}=0.3$. The reinforcement volume fraction and the interphase parameter are $V_{2}=0.4$ and $\eta=0.001$, respectively. This figure illustrates the comparisons between the composite effective properties with interphase computed by AHM and FEM using the spring-capacitor model 

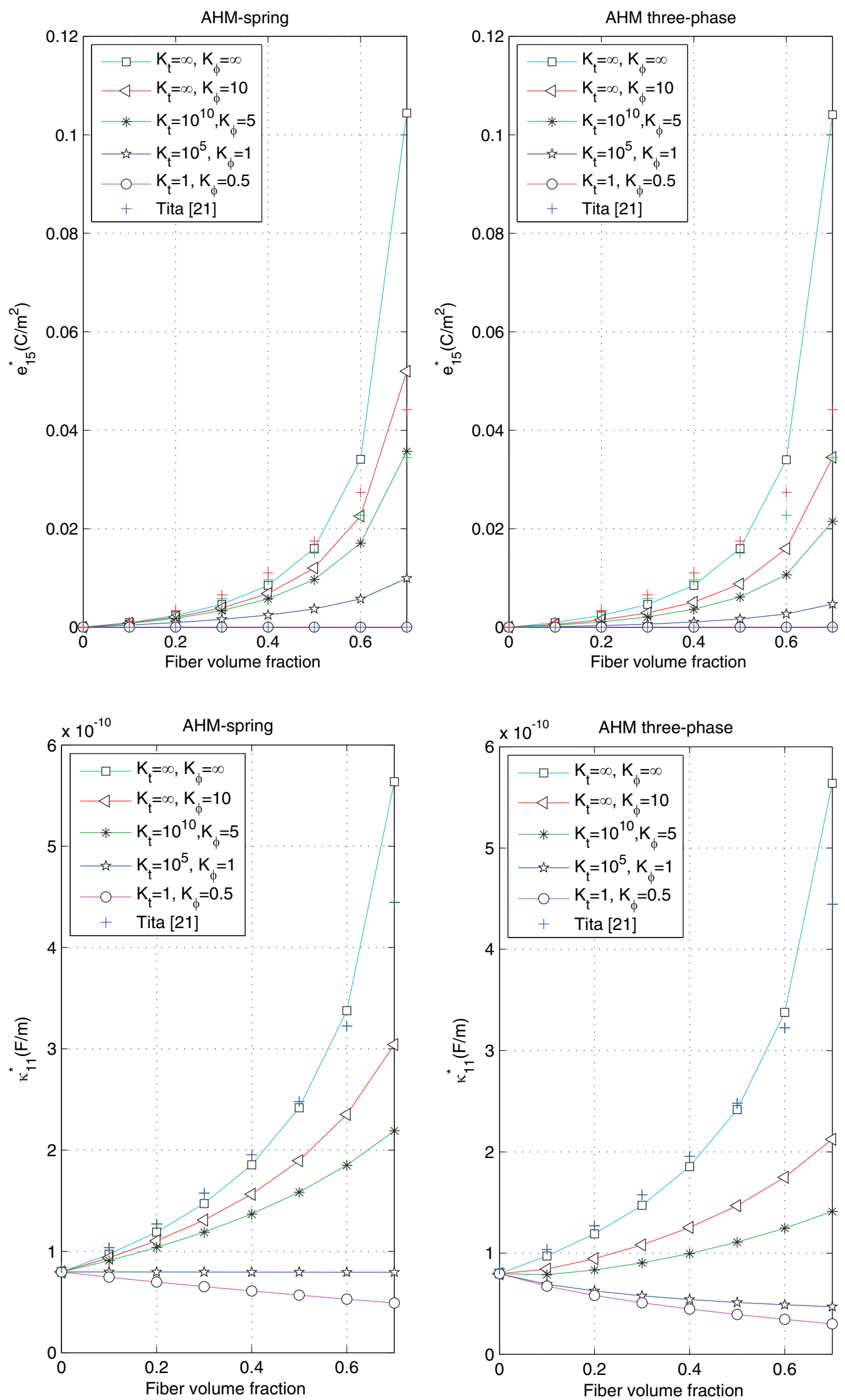

Figure 7. Evolution of the transversal piezoelectric and dielectric permittivity effective coefficients $\mathrm{e}_{15}^{*}$ and $\kappa_{1 \mid}^{*}$ for different values of the imperfect electric parameter $K_{\phi}$. 

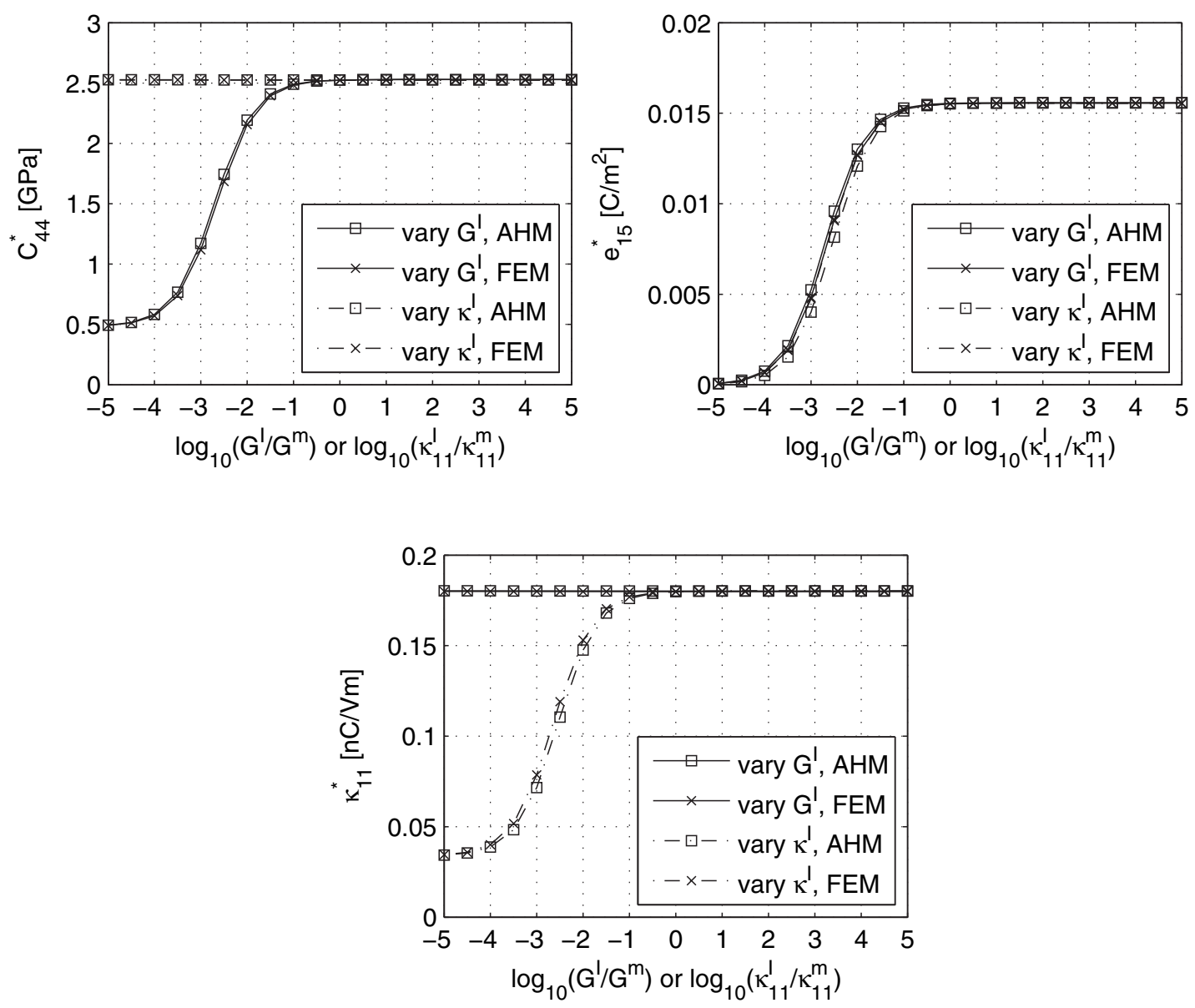

Figure 8. Comparison of effective coefficients $C_{44}^{*}, e_{15}^{*}$ and $\kappa_{11}^{*}$ between AHM and FEM by varying ratios of $G^{l} \equiv G^{l} / G^{m}$ or $\kappa^{\prime} \equiv \kappa_{11}^{l} / \kappa_{11}^{m}$.

for two cases. (a) Varying the elastic imperfect parameters $\left(\tilde{K}_{i}, i=r, \vartheta, z\right)$ by changing the ratio $G^{I} / G^{m}$ while the electrical imperfect parameter $\left(\tilde{K}_{\phi}\right)$ is constant with $\kappa_{11}^{I} / \kappa_{11}^{m}=1$ (solid lines in Figure 8); conversely (b) Elastic imperfect parameters are constant with $G^{I} / G^{m}=1$ and the electrical imperfect parameter $\left(\tilde{K}_{\phi}\right)$ varies by changing $\kappa_{11}^{I} / \kappa_{11}^{m}$ (dashed lines in Figure 8). Considering FEM the relation between the ratio and the imperfect parameters are given by equations (43) and (44). From a fixed ratio $G^{I}$ or $\kappa^{I}$ and using equation (43) or (44) the contact parameters are determined. The continuous lines correspond to variation of $G^{I}$ and discontinuous lines to variation of $\kappa^{I}$. The following tendencies can be observed. In case (a) the mechanical imperfection does not affect the dielectric permittivity $\kappa_{11}$ which is always constant, but $e_{15}^{*}$ and $C_{44}^{*}$ change considerably. However, case (b) illustrates that $C_{44}^{*}$ is not affected whereas $e_{15}$ and $\kappa_{11}$ vary. Furthermore, the highest gradient fluctuates are in the interval of considering ratio from -4 to -1 because the interface property is softer than the matrix.
Figure 9 displays the behavior of the effective coefficients $C_{44}^{*}, \quad e_{15}^{*}$ and $\kappa_{11}^{*}$ against $\log _{10}\left(G^{I} / G^{m}\right)$ or $\log _{10}\left(\kappa_{11}^{I} / \kappa_{11}^{m}\right)$, respectively, related to same variation of both $K_{i}$ mechanical and $K_{\phi}$ electrical imperfect parameter using both AHM and FEM methods. In this situation it is remarkable the abrupt change of the three global properties in the composite.

\section{Conclusions}

In this work, three approaches are presented to study the mechanical and electrical imperfections in reinforced piezoelectric composite materials with unidirectional cylindrical fibers periodically distributed in a rhombic cell pattern. Three-phase model is developed where the third phase is the interphase between the matrix and the fiber with certain thickness and also the spring-capacitor model is also proposed. This gives the possibility to study in a more realistic way the imperfect bonding between the phases for piezoelectric composites. Two special types of imperfections are considered using springs for mechanical contact and 

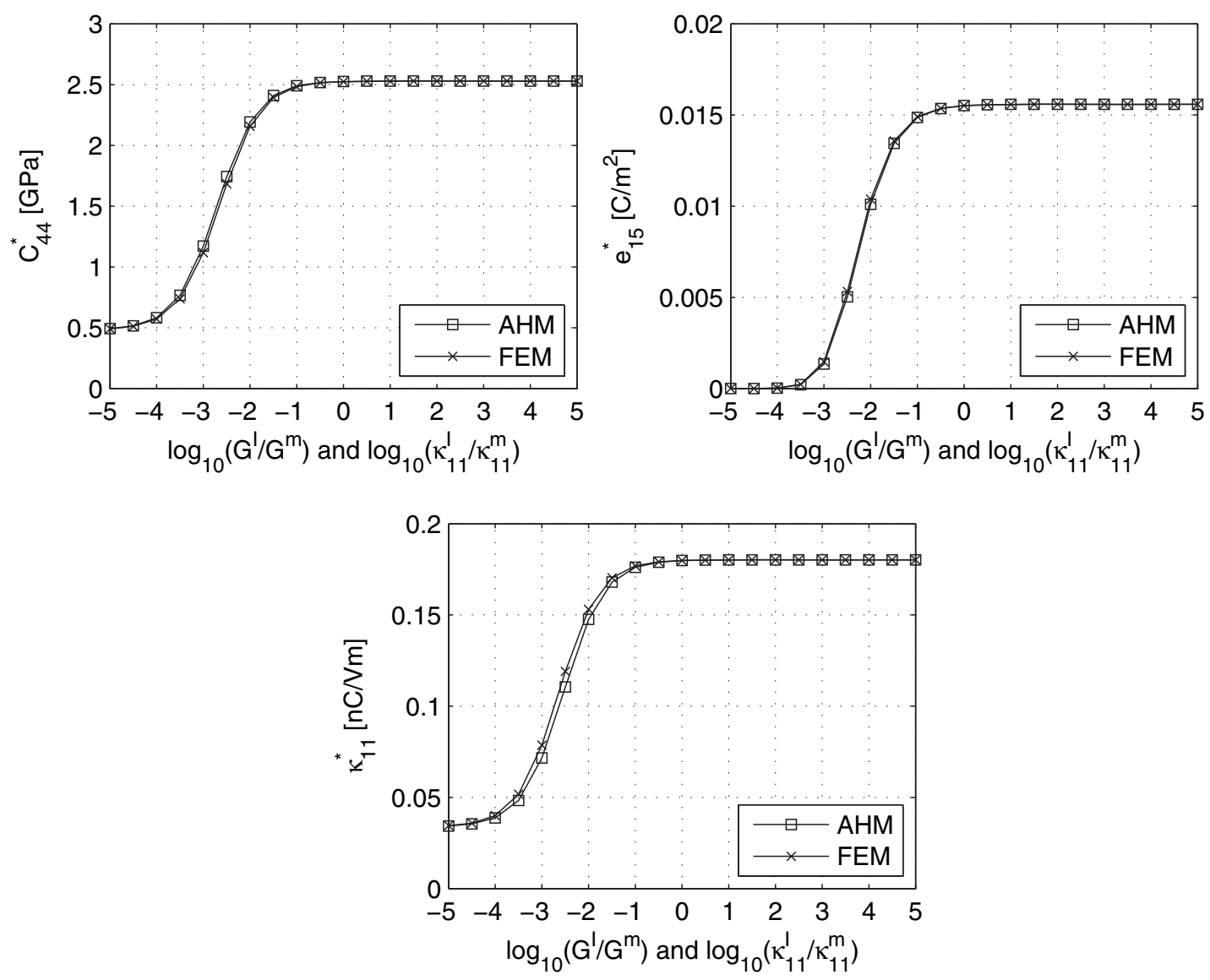

Figure 9. Comparison of effective coefficients $C_{44}^{*}, \mathrm{e}_{15}^{*}$ and $\kappa_{1,}^{*}$ between AHM and FEM by varying ratios of $G^{l} \equiv G^{l} / G^{m}$ and $\kappa^{l} \equiv \kappa_{1 \mid}^{l} / \kappa_{||}^{m}$ in the same manner.

capacitors for the electrical one, both are coupled. The spring-capacitor and three-phase models are implemented using AHM and FEM is developed for the spring-capacitor at the interface. The models reflect the influence of the mechanical and electrical imperfection on the behavior of the electro-mechanical effective coefficients. The present method can provide benchmark results for other numerical and approximate methods, and they may be useful in the computer-aid design of new materials.

\section{Acknowledgements}

The authors gratefully acknowledge Departamento de Matemáticas y Mecánica, IIMAS-UNAM, for its support and Ramiro Chávez Tovar and Ana Pérez Arteaga for their computer support. The four first authors RRR, RGD, JCLR and JBC render honor to Professor Sabina on his 70th anniversary.

\section{Funding}

The authors gratefully acknowledge the SHICHAN project, supported by FSP (Cooperation Scientifique FrancoCubaine) Cuba 2011-26, PROJET N²9935XH. The funding of CONACYT project number 129658 and Coordinación de la Investigación Cientifica (COIC-STIA-306-15 (7178-7179)) de la UNAM is gratefully acknowledged.

\section{Conflict of interest}

None declared.

\section{References}

1. Andrianov IV, Bolshakov VI, Danishevs'kyy VV, et al. Asymptotic simulation of imperfect bonding in periodic fibre-reinforced composite materials under axial shear. Int J Mech Sci 2007; 49: 1344-1354.

2. Andrianov IV, Bolshakov VI, Danishevs'kyy VV, et al. Asymptotic study of imperfect interfaces in conduction through a granular composite material. Proc $R$ Soc $A$ 2010; 466: 2707-2725.

3. Benveniste Y. The effective mechanical behavior of a composite with imperfect contact between the constituents. Mech Mater 1985; 4: 197-208.

4. Benveniste $\mathrm{Y}$ and Miloh T. The effective conductivity of composites with imperfect thermal contact at constituent interfaces. Int J Eng Sci 1986; 24: 1537-1552. 
5. Benveniste $\mathrm{Y}$ and Miloh T. Imperfect soft and stiff interfaces in two dimensional elasticity. Mech Mater 2001; 33: 309-323.

6. Jasiuk I and Tong T. The effect of interface on the elastic stiffness of composites. Mech Comput Mat Struct 1989; 100: 49-54.

7. Hashin Z. Thermoelastic properties of fiber composites with imperfect interface. Mech Mater 1990; 8: 333-348.

8. Bigoni D, Serkov SK, Valentini M, et al. Asymptotic models of dilute composites with imperfectly bonded inclusions. Int J Solids Struct 1998; 35: 3239-3258.

9. Kamali MT and Shodja HM. A semi-analytical method for piezocomposite structures with arbitrary interfaces. Comput Methods Appl Mech Engrg 2005; 194: 4588-4604.

10. Shodja HM, Tabatabaei SM and Kamali MT. A piezoelectric-inhomogeneity system with imperfect interface. Int J Eng Sci 2006; 44: 291-311.

11. Shodja HM, Tabatabaei SM and Kamali MT. A piezoelectric medium containing a cylindrical inhomogeneity: Role of electric capacitors and mechanical imperfections. Int J Solids Struct 2007; 44: 6361-6381.

12. Hashemi R, Weng GJ, Kargarnovin $\mathrm{MH}$, et al. Piezoelectric composites with periodic multi-coated inhomogeneities. Int J Solids Struct 2010; 47: 2893-2904.

13. Sanchez-Palencia E. Non homogeneous media and vibration theory lectures notes in physics. Berlin: Springer, 1980.

14. Pobedria BE. Mechanics of composite materials (in Russian). Moscow: Moscow State University Press, 1984.

15. Bakhvalov NS and Panasenko GP. Homogenization averaging processes in periodic media. Dordrecht, The Netherlands: Kluwer Academic Publishers, 1989.

16. Rodríguez-Ramos R, Guinovart-Diaz R, López JC, et al. Influence of imperfect elastic contact condition on the antiplane effective properties of piezoelectric fibrous composites. Arch Appl Mech 2010; 80: 377-388.

17. Rodríguez-Ramos R, Guinovart-Díaz R, LópezRealpozo JC, et al. Effective properties of periodic fibrous electro-elastic composites with mechanic imperfect contact condition. Int J Mech Sci 2013; 73: 1-13.

18. Rodríguez-Ramos R, Guinovart-Díaz R, LópezRealpozo JC, et al. Micromechanical analysis of fibrous piezoelectric composites with imperfectly bonded adherences. Arch Appl Mech 2014; 84: 1565-1582.

19. Rodríguez-Ramos R, López-Realpozo J, Guinovart-Díaz $\mathrm{R}$, et al. Micromechanical characterization of the effective properties for angular piezoelectric fibrous composites with imperfect contact condition. In: Proceedings of 11th Pan-American Congress of Applied Mechanics (PACAM XI). 04-08 January 2010, Foz do Iguaçu, PR, Brazil.

20. Hashin Z. The spherical inclusion with imperfect interface. J Appl Mech 1991; 58: 444-449.

21. Tita V, Medeiros de R, Marques FD, et al. Effective properties evaluation for smart composite materials with imperfect fiber-matrix adhesion. J Comp Mat 2015; 1: 1-19.

22. Bensoussan A, Lions JL and Papanicolaou G. Asymptotic analysis for periodic structures. New York; Amsterdam: North-Holland, 1978.
23. Bravo-Castillero J, Guinovart Díaz R, Sabina FJ, et al. Closed-form expressions for the effective coefficients of a fiber-reinforced composite with transversely isotropic constituents-II. Piezoelectric and square symmetry. Mech Mater 2001; 33: 237-248.

24. Royer D and Dieulesaint E. Elastic waves in solids I. Berlin, Heidelberg: Springer Verlag, 2000.

25. Sabina FJ, Rodríguez Ramos R, Bravo Castillero J, et al. Closed-form expressions for the effective coefficients of fibre-reinforced composite with transversely isotropic constituents-II: piezoelectric and hexagonal symmetry. J Mech Phys Solids 2001; 49: 1463-1479.

26. Chih-Bing L. Tables of values of relating to Weierstrass' elliptic function. Math Comput 1965; 19: 123-127.

27. Guinovart-Díaz R, Yan P, Rodríguez-Ramos R, et al. Effective properties of piezoelectric composites with parallelogram periodic cells. Int J Eng Sci 2012; 53: 58-66.

28. Guinovart-Díaz R, Rodriguez-Ramos R, BravoCastillero $\mathrm{J}$, et al. Closed-form thermo-elastic moduli of a periodic three-phase fiber-reinforced composite. $J$ Therm Str 2005; 28: 1067-1093.

29. Hashin Z. Thin interphase/imperfect interface in elasticity with application to coated fiber composites. $J$ Mech Phys Solids 2002; 50: 2509-2537.

30. Yan P, Jiang CP and Song F. An eigenfunction expansion-variational method for the anti-plane electroelastic behavior of three-phase fiber composites. Mech Mater 2011; 43: 586-597.

31. Pastor J. Homogenization of linear piezoelectric media. Mech Res Commun 1997; 24: 145-150.

32. Pettermann HE and Suresh S. A comprehensive unit cell model: a study of coupled effects in piezoelectric 1-3 composites. Int J Solids Struct 2000; 37: 5447-5464.

33. Berger H, Kari S, Gabbert U, et al. Unit cell models of piezoelectric fiber composites for numerical and analytical calculation of effective properties. Smart Mater Struct 2006; 15: 451-458.

34. Würkner M, Berger H and Gabbert U. Numerical study of effective elastic properties of fiber reinforced composites with rhombic cell arrangements and imperfect interface. Int J Eng Sci 2013; 63: 1-9.

\section{Appendix I}

Substituting expansions (20) and (33) into contact conditions (30) we obtain the following equations

$$
\begin{aligned}
& {\left[a_{0} \delta_{1 p}+\sum_{k=1}^{\infty}{ }^{*} a_{k} \eta_{k p}+\bar{a}_{p}\right] R^{p}=a_{p}^{(I)} R_{1}^{p}+\bar{a}_{-p}^{(I)} R^{p}} \\
& {\left[b_{0} \delta_{1 p}+\sum_{k=1}^{\infty}{ }^{*} b_{k} \eta_{k p}+\bar{b}_{p}\right] R^{p}=\sqrt{\chi_{1} / \kappa_{1}}} \\
& \times\left(b_{p}^{(I)} R_{1}^{p}+\bar{b}_{-p}^{(I)} R^{p}\right) \text { on } \Gamma_{1}
\end{aligned}
$$




$$
\begin{aligned}
a_{p}^{(I)} R^{p}+\bar{a}_{-p}^{(I)} R_{1}^{p} & =c_{p} R^{p} \\
\sqrt{\chi_{1} \kappa_{2}}\left[b_{p}^{(I)} R^{p}+\bar{b}_{-p}^{(I)} R_{1}^{p}\right] & =\sqrt{\kappa_{1} \chi_{2}} d_{p} R^{p} \quad \text { on } \Gamma_{2}, \\
\chi_{1} & =C_{1313}^{(I)} / C_{1313}^{(1)}, \chi_{2}=C_{1313}^{(2)} / C_{1313}^{(1)}, \\
\kappa_{1} & =\kappa_{11}^{(I)} / \kappa_{11}^{(1)}, \kappa_{2}=\kappa_{11}^{(2)} / \kappa_{11}^{(1)}, \\
E_{\beta}^{(s)} & =e_{\beta \beta 3}^{(s)} / \sqrt{C_{\beta 3 \beta 3}^{(s)} \kappa_{\beta \beta}^{(s)}}, \quad s=1, I, 2
\end{aligned}
$$

Now, the local functions are considered as periodic harmonic functions of the complex variable, which satisfy the Cauchy-Riemann conditions, $\left[\left[f_{i}\right]\right]=f_{i}(z)-\bar{f}_{i}(z)$ and $n_{2}=-R^{-1}\left(d x_{1} / \mathrm{d} \theta\right), n_{1}=R^{-1}\left(d x_{2} / \mathrm{d} \theta\right)$ on the interfaces $\Gamma_{\gamma}$, the equalities (31) can be written in terms of the complex functions as

$$
\begin{aligned}
& {\left[\begin{array}{c}
{\left[\left[f_{1}\right]\right]+E_{15}^{(1)}\left[\left[g_{1}\right]\right]+\left(1-\chi_{1}\right) \delta_{\alpha 1}(z-\bar{z})=} \\
=\chi_{1}\left[\left[\left[f_{2}\right]\right]+E_{15}^{(I)}\left[\left[g_{2}\right]\right]\right]+\left(1-\chi_{1}\right) i \delta_{\alpha 2}(z+\bar{z})
\end{array}\right] \|_{\Gamma_{1}}} \\
& {\left[\begin{array}{c}
\chi_{1}\left[\left[\left[f_{2}\right]\right]+E_{15}^{(I)}\left[\left[g_{2}\right]\right]\right]+\left(\chi_{2}-\chi_{1}\right) i \delta_{\alpha 2}(z+\bar{z})= \\
=\left(\chi_{2}-\chi_{1}\right) \delta_{\alpha 1}(z-\bar{z})+\chi_{2}\left[\left[\left[f_{3}\right]\right]+E_{15}^{(2)}\left[\left[g_{3}\right]\right]\right]
\end{array}\right] \|_{\Gamma_{2}}}
\end{aligned}
$$

$$
\begin{aligned}
& \chi_{1}\left\{\left[a_{p}^{(I)}+E_{15}^{(I)} b_{p}^{(I)}+Q_{15}^{(I)} e_{p}^{(I)}\right] R^{p}-\left[\bar{a}_{-p}^{(I)}+E_{15}^{(I)} \bar{b}_{-p}^{(I)}\right] R_{1}^{p}\right\} \\
& =\chi_{2}\left[c_{p}+E_{15}^{(2)} d_{p}\right] R^{p}+\left(\chi_{2}-\chi_{1}\right) R^{p+1}\left[\delta_{\alpha 1}-i \delta_{\alpha 2}\right] \delta_{1 p} \\
& \left\{E_{15}^{(1)}\left[a_{0} \delta_{1 p}+\sum_{k=1}^{\infty}{ }^{*} a_{k} \eta_{k p}-\bar{a}_{p}\right]\right. \\
& \left.-\left[b_{0} \delta_{1 p}+\sum_{k=1}^{\infty} b_{k} \eta_{k p}-\bar{b}_{p}\right]\right\} R^{p} \\
& =\sqrt{\chi_{1} \kappa_{1}}\left\{\left[E_{15}^{(I)} a_{p}^{(I)}-b_{p}^{(I)}\right] R_{1}^{p}-\left[E_{15}^{(I)} \bar{a}_{-p}^{(I)}-\bar{b}_{-p}^{(I)}\right] R^{p}\right\} \\
& -\left(E_{15}^{(1)}-\sqrt{\chi_{1} \kappa_{1}} E_{15}^{(I)}\right) R^{p} R_{1} \delta_{1 p}\left[\delta_{\alpha 1}-i \delta_{\alpha 2}\right], \\
& \sqrt{\chi_{1} \kappa_{1}}\left\{\left[E_{15}^{(I)} a_{p}^{(I)}-b_{p}^{(I)}\right] R^{p}-\left[E_{15}^{(I)} \bar{a}_{-p}^{(I)}-\bar{b}_{-p}^{(I)}\right] R_{1}^{p}\right\} \\
& =\sqrt{\chi_{2} \kappa_{2}}\left[E_{15}^{(2)} c_{p}-d_{p}\right] R^{p}-\left(\sqrt{\chi_{1} \kappa_{1}} E_{15}^{(I)}-\sqrt{\chi_{2} \kappa_{2}} E_{15}^{(2)}\right) \\
& \quad \times R^{p+1} \delta_{1 p}\left[\delta_{\alpha 1}-i \delta_{\alpha 2}\right]
\end{aligned}
$$

We have obtained a system (57), (58), (61) and (62) of eight equations with the unknown constants $\bar{a}_{p}, a_{p}^{(I)}, \bar{a}_{-p}^{(I)}, \bar{b}_{p}, b_{p}^{(I)}, \bar{b}_{-p}^{(I)}, \quad c_{p}$ and $d_{p}$. This system

$$
\begin{aligned}
& {\left.\left[\begin{array}{l}
E_{15}^{(1)}\left[\left[f_{1}\right]\right]-\left[\left[g_{1}\right]\right]+\left(E_{15}^{(1)}-\sqrt{\chi_{1} \kappa_{1}} E_{15}^{(I)}\right)(z-\bar{z}) \delta_{\alpha 1}= \\
=\sqrt{\chi_{1} \kappa_{1}}\left[E_{15}^{(I)}\left[\left[f_{2}\right]\right]-\left[\left[g_{2}\right]\right]\right]+i\left(E_{15}^{(1)}-\sqrt{\chi_{1} \kappa_{1}} E_{15}^{(I)}\right)(z+\bar{z}) \delta_{\alpha 2}
\end{array}\right]\right|_{\Gamma_{1}}} \\
& {\left.\left[\begin{array}{l}
\sqrt{\chi_{1} \kappa_{1}}\left[E_{15}^{(I)}\left[\left[f_{2}\right]\right]-\left[\left[g_{2}\right]\right]\right]+\left(\sqrt{\chi_{1} \kappa_{1}} E_{15}^{(I)}-\sqrt{\chi_{2} \kappa_{2}} E_{15}^{(2)}\right)(z-\bar{z}) \delta_{\alpha 1}= \\
=\sqrt{\chi_{2} \kappa_{2}}\left[E_{15}^{(2)}\left[\left[f_{3}\right]\right]-\left[\left[g_{2}\right]\right]\right]+i\left(\sqrt{\chi_{1} \kappa_{1}} E_{15}^{(I)}-\sqrt{\chi_{2} \kappa_{2}} E_{15}^{(2)}\right) \delta_{\alpha 2}(z+\bar{z})
\end{array}\right]\right|_{\Gamma_{2}}}
\end{aligned}
$$

Then substituting the expansion (20) and (33) into equations (59) - (60) at the interface $\Gamma_{s}$ and proceeding in an analogous form for obtaining (57) - (58), the following equations characterize the contact conditions.

$$
\begin{aligned}
& \left\{\left[a_{0} \delta_{1 p}+\sum_{k=1}^{\infty}{ }^{*} a_{k} \eta_{k p}-\bar{a}_{p}\right]\right. \\
& \left.\quad+E_{15}^{(1)}\left[b_{0} \delta_{1 p}+\sum_{k=1}^{\infty}{ }^{*} b_{k} \eta_{k p}-\bar{b}_{p}\right]\right\} R^{p} \\
& =\chi_{1}\left\{\left[a_{p}^{(I)}+E_{15}^{(I)} b_{p}^{(I)}\right] R_{1}^{p}-\left[\bar{a}_{-p}^{(I)}+E_{15}^{(I)} \bar{b}_{-p}^{(I)}\right] R^{p}\right\} \\
& \quad-\left(1-\chi_{1}\right) R^{p} R_{1}\left[\delta_{\alpha 1}-i \delta_{\alpha 2}\right] \delta_{1 p}
\end{aligned}
$$

can be solved by Gauss's method. Once we have the unknown constants, we can determine the effective coefficients (36)-(39). 